\title{
Spatial degrees-of-freedom in large-array full-duplex: the impact of backscattering
}

\author{
Evan Everett ${ }^{*}$ and Ashutosh Sabharwal
}

\begin{abstract}
The key challenge for in-band full-duplex wireless communication is managing self-interference. Many designs have employed spatial isolation mechanisms, such as shielding or multi-antenna beamforming, to isolate the self-interference waveform from the receiver. Because such spatial isolation methods confine the transmit and receive signals to a subset of the available space, the full spatial resources of the channel may be under-utilized, expending a cost that may nullify the net benefit of operating in full-duplex mode. In this paper, we leverage an antenna-theory-based channel model to analyze the spatial degrees of freedom available to a full-duplex capable base station. We observe that whether or not spatial isolation out-performs time-division (i.e., half-duplex) depends heavily on the geometric distribution of scatterers. Unless the angular spread of the objects that scatter to the intended users is overlapped by the spread of objects that backscatter to the base station, then spatial isolation outperforms time division, otherwise time division may be optimal.
\end{abstract}

\section{Introduction}

Currently deployed wireless communications equipment operates in half-duplex mode, meaning that transmission and reception are orthogonalized either in time (time-division-duplex) or frequency (frequency-divisionduplex). Research in recent years [1-12] has investigated the possibility of wireless equipment operating in fullduplex mode, meaning that the transceiver will both transmit and receive at the same time and in the same spectrum. A potential benefit of full-duplex is illustrated in. User 1 wishes to transmit uplink data to a base station, and User 2 wishes to receive downlink data from the same base station. If the base station is half-duplex, then it must either service the users in orthogonal time slots or in orthogonal frequency bands. However, if the base station can operate in full-duplex mode, then it can enhance spectral efficiency by servicing both users simultaneously. The challenge to full-duplex communication, however, is that the base station transmitter generates high-powered selfinterference which potentially swamps its own receiver, precluding the detection of the uplink message. ${ }^{1}$

*Correspondence: evan.everett@rice.edu

This paper was presented in part at the 2014 IEEE International Symposium on Information Theory.

Department of Electrical and Computer Engineering, Rice University, Houston, TX 77005, USA
For full-duplex to be feasible, the self-interference must be suppressed. The two main approaches to selfinterference suppression are cancellation and spatial isolation, and we now define each. Self-interference cancellation is any technique which exploits the foreknowledge of the transmit signal by subtracting an estimate of the self-interference from the received signal. Cancellation can be applied at digital baseband, at analog baseband, at RF, or, as is most common, applied at a combination of these three domains $[4-7,11,13,14]$. Spatial isolation is any technique to spatially orthogonalize the selfinterference and the signal-of-interest. Some spatial isolation techniques studied in the literature are multi-antenna beamforming [1, 15-19], directional antennas [20], shielding via absorptive materials [21], and cross-polarization of transmit and receive antennas [10,21]. The key differentiator between cancellation and spatial isolation is that cancellation requires and exploits knowledge of the self-interference, while spatial isolation does not. To our knowledge, all full-duplex designs to date have required both cancellation and spatial isolation in order for fullduplex to be feasible even at very short ranges (i.e., $<10$ $\mathrm{m})$. For example, see designs such as $[5,6,10,11]$, each of which leverages cancellation techniques as well as at least one spatial isolation technique. Moreover, because cancellation performance is limited by transceiver impairments such as phase noise [22], spatial isolation often accounts 
for an outsized portion of the overall self-interference suppression.

For example, in the full-duplex design of [21] which demonstrated full-duplex feasibility at WiFi ranges, of the $95 \mathrm{~dB}$ of self-interference suppression achieved, $70 \mathrm{~dB}$ is due to spatial isolation, while only $25 \mathrm{~dB}$ is due to cancellation. Therefore, if full-duplex feasibility is to be extended from WiFi-typical ranges to the ranges typical of femtocells or even larger cells, then excellent spatial isolation performance will be required, hence our focus is on spatial isolation in this paper.

In a previous work [21], we studied three passive techniques for spatial isolation: directional antennas, absorptive shielding, and cross-polarization, and measured their performance in a prototype base station both in an anechoic chamber that mimics free space, and in a reflective room. As expected, the techniques suppressed the self-interference quite well (more than $70 \mathrm{~dB}$ ) in an anechoic chamber, but scattering environments, the suppression was much less, (no more than $45 \mathrm{~dB}$ ), due to the fact that passive techniques operate primarily on the direct path between the transmit and receive antennas, and do little to suppress paths that include an external backscatterer. The direct-path limitation of passive spatial isolation mechanisms raises the question of whether or not spatial isolation can be useful in a backscattering environment. Another class of spatial isolation techniques called "active" or "channel aware" spatial isolation [23] can indeed suppress both direct and backscattered self-interference. In particular, if multiple antennas are used and if the self-interference channel response can be estimated, then the radiation pattern can be shaped adaptively to mitigate both direct-path and backscattered self-interference. However, this pattern shaping (i.e., beamforming) will consume spatial degrees-of-freedom that could have otherwise been leveraged for spatial multiplexing. Thus, there is an important tradeoff between spatial self-interference isolation and achievable degrees of freedom.

To appreciate the tradeoff, consider the example in Fig. 1. The direct path from the base station transmitter, $T_{2}$, to its receiver $R_{1}$, can be passively suppressed by shielding the receiver from the transmitter as shown in [21], but there will also be backscattered self-interference due to objects near the base station (depicted by gray blocks in Fig. 1). The self-interference caused by scatterer $S_{0}$, for example, in Fig. 1, could be avoided by creating a null in the direction of $S_{0}$. However, losing access to the scatterer could create a less-rich scattering environment, diminishing the spatial degrees-of-freedom of the uplink or downlink. Moreover, creating the null consumes spatial degrees-of-freedom that could otherwise be used for spatial multiplexing to the downlink user, diminishing the achievable degrees-of-freedom of

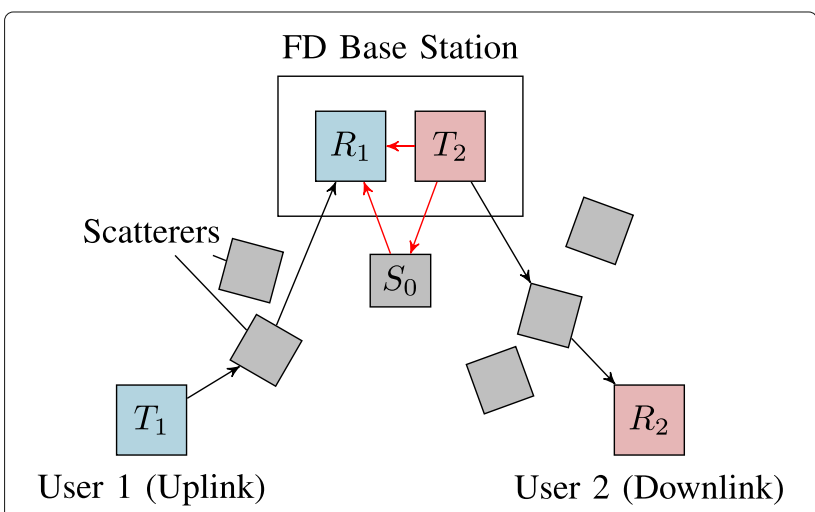

Fig. 1 Three-node full-duplex model

the downlink. This example leads us to pose the following question.

Question: Under what scattering conditions can spatial isolation be leveraged in full-duplex operation to provide a degree-of-freedom gain over half-duplex? More specifically, given a constraint on the size of the antenna arrays at the base station and at the user devices, and given a characterization of the spatial distribution of the scatterers in the environment, what is the uplink/downlink degree-offreedom region when the only self-interference mitigation strategy is spatial isolation?

Modeling approach: To answer the above question, we leverage the antenna-theory-based channel model developed by Poon, Broderson, and Tse in [24-26], which we will label the "PBT" model. In the PBT model, instead of constraining the number of antennas, the size of the array is constrained. Furthermore, instead of considering a channel matrix drawn from a probability distribution, a channel transfer function which depends on the geometric position of the scatterers relative to the arrays is considered (Fig. 2).

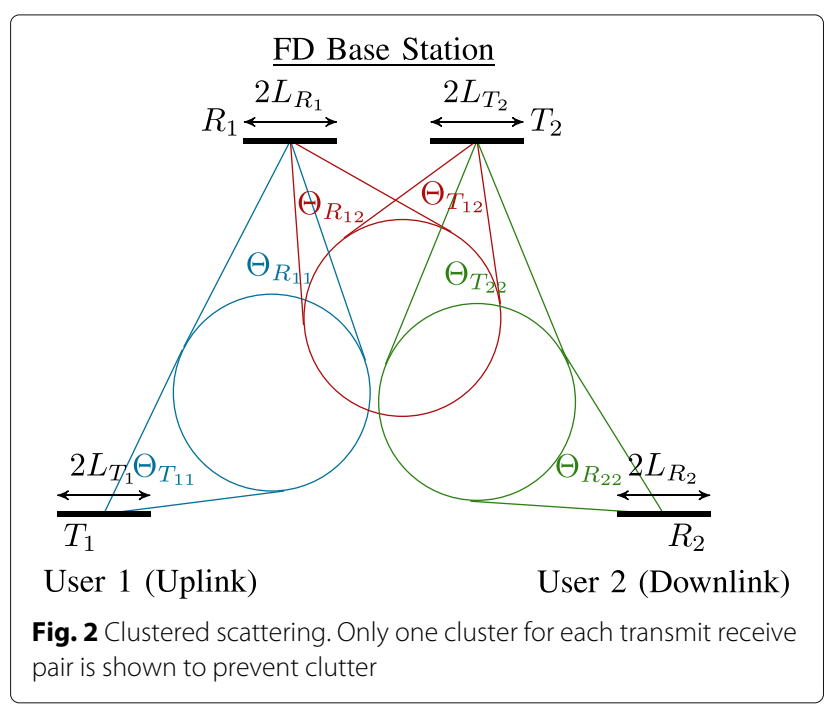


Contribution: We extend the PBT model to the threenode full-duplex topology of Fig. 1, and derive the degree-of-freedom region, $\mathcal{D}_{\mathrm{FD}}$ : the set of all achievable uplink/downlink degree-of-freedom tuples. By comparing $\mathcal{D}_{\mathrm{FD}}$ to $\mathcal{D}_{\mathrm{HD}}$, the degree-of-freedom region achieved by time-division half-duplex, we observe that full-duplex outperforms half-duplex, i.e., $\mathcal{D}_{\mathrm{HD}} \subset \mathcal{D}_{\mathrm{FD}}$, in the following two scenarios.

1. When the base station arrays are larger than the corresponding user arrays, the base station has a larger signal space than is needed for spatial multiplexing and can leverage the extra signal dimensions to form beams that avoid self-interference (i.e., self-interference zero-forcing).

2. More interestingly, when the forward scattering intervals and the backscattering intervals are not completely overlapped, the base station can avoid self-interference by signaling in the directions that scatter to the intended receiver, but do not backscatter to the base-station receiver. Moreover, the base station can also signal in directions that do cause self-interference, but ensure that the generated self-interference is incident on the base-station receiver only in directions in which uplink signal is not incident on the base-station receiver, i.e., signal such that the self-interference and uplink signal are spatially orthogonal.

In [27], an experimental evaluation of a transmitbeamforming-based method for full-duplex operation called "SoftNull" is presented. Inspired by the achievability proof in Section 3.1, the SoftNull algorithm presented in [27] seeks to maximally suppress self-interference for a given required number of downlink-degrees-of-freedom. This paper presents an information theoretic analysis of the performance limits of beamforming-based full-duplex systems, whereas [27] presents an experimental evaluation of a specific design. We would like to refer [27] to readers who may be interested in how the theoretical intuitions from this paper can guide the design and implementation of a beamforming-based full-duplex system.

Organization of the paper: Section 2 specifies the system model: we begin with an overview of the PBT model in Section 2.1 and then in Section 2.2 apply the model to the scenario of a full-duplex base station with uplink and downlink flows. Section 3 gives the main analysis of the paper, the derivation of the degrees-offreedom region. We start Section 3 by stating the theorem which characterizes the degrees-of-freedom region and then give the achievability and converse arguments in Sections 3.1 and 3.2, respectively. In Section 4, we assess the impact of the degrees-of-freedom result on the design and deployment of full-duplex base stations, and include an application example, that shows how the results of this paper are used to guide the design of a full-duplex base station in [27]. We give concluding remarks in Section 5.

\section{System model}

We now give a brief overview of the PBT channel model presented in [24]. We then extend the PBT model to the case of the three-node full-duplex topology of Fig. 1, and define the required mathematical formalism that will ease the degrees-of-freedom analysis in the sequel.

\subsection{Overview of the PBT model}

As illustrated in Fig. 3, the PBT channel model considers a wireless communication link between a transmitter equipped with a unipolarized continuous linear array of length $2 L_{T}$ and a receiver with a similar array of length $2 L_{R}$. The authors observe that there are two key domains: the array domain, which describes the current distribution on the arrays, and the wavevector domain which describes radiated and received field patterns. Channel measurement campaigns show that the angles of departure and the angles of arrival of the physical paths from a transmitter to a receiver tend to be concentrated within a handful of angular clusters [28-31]. Thus the authors of the PBT model [24] focus on the union of the clusters of angles-of-departure from the transmit array, denoted $\Theta_{T}$, and the union of the clusters of angles-of-arrival to the receive array, $\Theta_{R}$. Because a linear array aligned to the $z$-axis array can only resolve the $z$-component, the intervals of interest are $\Psi_{T}=\left\{\cos \theta: \theta \in \Theta_{T}\right\}$ and $\Psi_{R}=\left\{\cos \theta: \theta \in \Theta_{R}\right\}$. In [24], it is shown from the first principles of Maxwell's equations that an array of length $2 L_{T}$ has a resolution of $1 /\left(2 L_{T}\right)$ over the interval $\Psi_{T}$, so that the dimension of the transmit signal space of radiated field patterns is $2 L_{T}\left|\Psi_{T}\right|$. Likewise the dimension of the receive signal space is $2 L_{R}\left|\Psi_{R}\right|$, so that the spatial degrees of freedom a point-to-point communication link, $d_{\mathrm{P} 2 \mathrm{P}}$, is

$$
d_{\mathrm{P} 2 \mathrm{P}}=\min \left\{2 L_{T}\left|\Psi_{T}\right|, 2 L_{R}\left|\Psi_{R}\right|\right\} .
$$

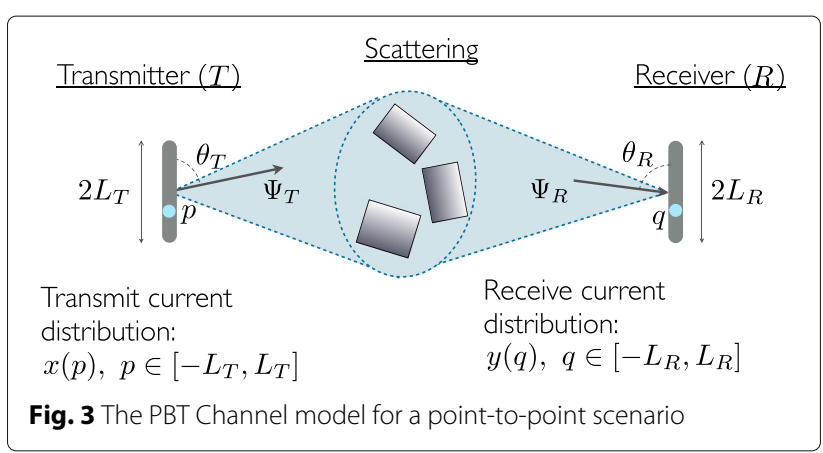




\subsection{Extension of PBT model to three-node full-duplex}

Now we extend the PBT channel model in [24], which considers a point-to-point topology, to the three-node full-duplex topology of Fig. 1. The antenna-theory-based PBT channel model is built upon far-field assumptions, i.e., that the propagation path is much larger than a wavelength. We acknowledge that direct-path self-interference may not obey far-field behavior. However, the backscattered self-interference, which will travel several wavelengths to reach an external scatterer and then return to the base station, is indeed a far-field signal. As discussed in the introduction, the intent of this paper is to understand the impact of backscattered self-interference as a function of the size of antenna arrays and the geometric distribution of the scatterers. Since the backscattering is indeed a far-field phenomenon, the PBT model is a quite well-suited model for our study.

As in [24], we consider continuous linear arrays of infinitely many infinitesimally small unipolarized antenna elements. $^{2}$ Each of the two transmitters $T_{j}, j=1,2$, is equipped with a linear array of length $2 L_{T_{j}}$, and each receiver, $R_{i}, i=1,2$, is equipped with a linear array of length $2 L_{R_{i}}$. The lengths $L_{T_{j}}$ and $L_{R_{i}}$ are normalized by the wavelength of the carrier, and thus are unitless quantities. For each array, define a local coordinate system with origin at the midpoint of the array and $z$-axis aligned along the length of the array. Let $\theta_{T_{j}} \in[0, \pi)$ denote the elevation angle relative to the $T_{j}$ array, and let $\theta_{R_{i}}$ denote the elevation angle relative to the $R_{i}$ array. Denote the current distribution on the $T_{j}$ array as $x_{j}\left(p_{j}\right)$, where $p_{j} \in\left[-L_{T_{j}}, L_{T_{j}}\right]$ is the position along the lengths of the array, and $x_{j}:\left[-L_{T_{j}}, L_{T_{j}}\right] \rightarrow \mathbb{C}$ gives the magnitude and phase of the current. The current distribution, $x_{j}\left(p_{j}\right)$, is the transmit signal controlled by $T_{j}$, which we constrain to be square integrable. Likewise, we denote the received current distribution on the $R_{i}$ array as $y_{i}\left(q_{i}\right), q_{i} \in$ $\left[-L_{R_{i}}, L_{R_{i}}\right]$.

The signal received by the base station receiver, $R_{1}$, at a point $q_{1} \in\left[-L_{R_{2}}, L_{R_{2}}\right]$ along its array is given by

$$
\begin{aligned}
y_{1}\left(q_{1}\right)= & \underbrace{\int_{-L_{T_{1}}}^{L_{T_{1}}} C_{11}\left(q_{1}, p_{1}\right) x_{1}\left(p_{1}\right) d p_{1}}_{\text {desired uplink signal }} \\
& +\underbrace{\int_{-L_{T_{2}}}^{L_{T_{2}}} C_{12}\left(q_{1}, p_{2}\right) x_{2}\left(p_{2}\right) d p_{2}}_{\text {self-interference }}+\underbrace{z_{1}\left(q_{1}\right),}_{\text {noise }}
\end{aligned}
$$

where $z_{1}\left(q_{1}\right), q_{1} \in\left[-L_{R_{1}}, L_{R_{1}}\right]$ is the noise along the $R_{1}$ array. The channel response integral kernel, $C_{i j}\left(q_{i}, p_{j}\right)$, gives the current excited at a point $q_{i}$ on the receive array due to a current at the point $p_{j}$ on the transmit array. Note that the first term in (2) gives the received uplink signal-of-interest, while the second term gives the selfinterference generated by the base station's own transmission. We assume that the mobile users are out of range of each other, such that there is no channel from $T_{1}$ to $R_{2}{ }^{3}$ Thus, $R_{2}$ 's received signal at a point $q_{2} \in\left[-L_{R_{2}}, L_{R_{2}}\right]$ is

$$
y_{2}\left(q_{2}\right)=\int_{-L_{T_{2}}}^{L_{T_{2}}} C_{22}\left(q_{2}, p_{2}\right) x_{2}\left(p_{2}\right) d p_{2}+z_{2}\left(q_{2}\right) \text {. }
$$

The channel response kernel, $C_{i j}(\cdot, \cdot)$ is composed of a transmit array response, $\boldsymbol{A}_{T_{j}}(\cdot, \cdot)$, a scattering response, $H_{i j}(\cdot, \cdot)$, and a receive array response, $\boldsymbol{A}_{R_{i}}(\cdot, \cdot)[24]$. The channel response kernel is given by

$$
\begin{aligned}
C_{i j}(q, p)= & \iint_{\text {Rx array response }}^{A_{R_{i}}(q, \hat{\boldsymbol{\kappa}})} \overbrace{H_{i j}(\hat{\boldsymbol{\kappa}}, \hat{\boldsymbol{k}})}^{\text {scattering response }} \\
& \times \underbrace{A_{T_{j}}(\hat{\boldsymbol{k}}, p)}_{\text {Tx array response }} d \hat{\boldsymbol{k}} d \hat{\boldsymbol{\kappa}},
\end{aligned}
$$

where $\hat{\boldsymbol{k}}$ is a unit vector that gives the direction of departure from the transmitter array, and $\hat{\kappa}$ is a unit vector that gives the direction of arrival to the receiver array. The transmit array response kernel, $\boldsymbol{A}_{T_{j}}(\hat{\boldsymbol{k}}, p)$, maps the current distribution along the $T_{j}$ array (a function of $p$ ) to the field pattern radiated from $T_{j}$ (a function of direction of departure, $\hat{\boldsymbol{k}})$. The scattering response kernel, $H_{i j}(\hat{\boldsymbol{k}}, \hat{\boldsymbol{k}})$, maps the fields radiated from $T_{j}$ in direction $\hat{\boldsymbol{k}}$ to the fields incident on $R_{i}$ at direction $\hat{\kappa}$. The receive array response, $A_{R_{i}}(q, \hat{\kappa})$, maps the field pattern incident on $R_{i}$ (a function of direction of arrival, $\hat{\kappa}$ ) to the current distribution excited on the $R_{i}$ array (a function of position $q$ ), which is the received signal.

\subsection{Array responses}

In [24], the transmit array response for a linear array is derived from the first principles of Maxwell's equations and shown to be $A_{T_{j}}(\hat{\boldsymbol{k}}, p)=A_{T_{j}}\left(\cos \theta_{T_{j}}, p\right)=$ $e^{-\mathrm{i} 2 \pi p \cos \theta_{T_{j}}}, p \in\left[-L_{T_{j}}, L_{T_{j}}\right]$, where $\theta_{T_{j}} \in[0, \pi)$ is the elevation angle relative to the $T_{j}$ array. Due to the symmetry of the array (aligned to the $z$-axis), the radiation pattern is symmetric with respect to the azimuth angle and only depends on the elevation angle $\theta_{T_{j}}$ through $\cos \theta_{T_{j}}$. For notational convenience, let $t \equiv \cos \theta_{T_{j}} \in[-1,1]$, so that we can simplify the transmit array response kernel to

$$
A_{T_{j}}(t, p)=e^{-\mathrm{i} 2 \pi p t}, t \in[-1,1], p \in\left[-L_{T_{j}}, L_{T_{j}}\right] .
$$

By reciprocity, the receive array response kernel, $A_{R_{i}}(q, \hat{\kappa})$, is

$$
A_{R_{i}}(q, \tau)=e^{\mathrm{i} 2 \pi q \tau}, \tau \in[-1,1], q \in\left[-L_{R_{i}}, L_{R_{i}}\right],
$$

where $\tau \equiv \cos \theta_{R_{i}} \in[-1,1]$ is the cosine of the elevation angle relative to the $R_{i}$ array. Note that the transmit 
and receive array response kernels are identical to the kernels of the Fourier transform and inverse Fourier transform, respectively, a relationship we will further explore in Section 2.5.

\subsection{Scattering responses}

The scattering response kernel, $H_{i j}(\hat{\boldsymbol{\kappa}}, \hat{\boldsymbol{k}})$, gives the amplitude and phase of the path departing from $T_{j}$ at direction $\hat{\boldsymbol{k}}$ and arriving at $R_{i}$ at direction $\hat{\boldsymbol{\kappa}}$. Since we are considering linear arrays which only resolve the cosine of the elevation angle, we can consider $H_{i j}(\tau, t)$ which gives the superposition of the amplitude and phase of all paths emanating from $T_{j}$ with an elevation angle whose cosine is $t$ and arriving at $R_{i}$ at an elevation angle whose cosine is $\tau$.

As is done in [24], motivated by measurements showing that scattering paths are clustered with respect to the transmitter and receiver, we adopt a model that focuses on the boundary of the scattering clusters rather than the discrete paths themselves, as illustrated in Fig. 2.

Let $\Theta_{T_{i j}}^{(k)}$ denote the angle subtended at transmitter $T_{j}$ by the $k^{\text {th }}$ cluster that scatters to $R_{i}$, and let $\Theta_{T_{i j}}=\bigcup_{k} \Theta_{T_{i j}}^{(k)}$ be the total transmit scattering interval from $T_{j}$ to $R_{i}$. This scattering interval, $\Theta_{T_{i j}}$, is the set of directions that when illuminated by $T_{j}$ scatters energy to $R_{i}$. In Fig. 2, to avoid clutter, we illustrate the case in which $\Theta_{T_{i j}}^{(k)}$ is a single contiguous angular interval, but in general, the interval will be non-contiguous and consist of several individual clusters. Similarly let $\Theta_{R_{i j}}^{(k)}$ denote the corresponding angle subtended at $R_{i}$ by the $k^{\text {th }}$ cluster illuminated by $T_{j}$, and let $\Theta_{R_{i j}}=\bigcup_{k} \Theta_{R_{i j}}^{(k)}$ be set of directions from which energy can be incident on $R_{i}$ from $T_{j}$.

Thus, we see in Fig. 2 that from the point-of-view of the base-station transmitter, $T_{2}, \Theta_{T_{22}}$ is the angular interval over which the base station can radiate signals that will reach the intended downlink receiver, $R_{2}$. The angular interval, $\Theta_{T_{12}}$, is the interval in which the base station's radiated signals will backscatter to the base station's own receiver, $R_{1}$, as self-interference. Likewise, from the pointof-view of the base station receiver, $R_{1}, \Theta_{R_{11}}$, is the interval over which the base station may receive signals from the uplink transmitter, $T_{1}$, while $\Theta_{R_{12}}$ is the interval in which self-interference may be present. Clearly, the extent to which the self-interference intervals and the signal-ofinterest intervals overlap will have a major impact on the degrees of freedom of the network. Because linear arrays can only resolve the cosine of the elevation angle $t \equiv$ $\cos \theta$, we define the "effective" scattering intervals for the transmit and receive arrays, respectively, as

$$
\begin{aligned}
& \Psi_{T_{i j}} \equiv\left\{t: \arccos (t) \in \Theta_{T_{i j}}\right\} \subset[-1,1], \\
& \Psi_{R_{i j}} \equiv\left\{\tau: \arccos (\tau) \in \Theta_{R_{i j}}\right\} \subset[-1,1] .
\end{aligned}
$$

Define the size of the transmit and receive scattering intervals as $\left|\Psi_{T_{i j}}\right|=\int_{\Psi_{T_{i j}}} t d t$ and $\left|\Psi_{R_{i j}}\right|=\int_{\Psi_{R_{i j}}} \tau d \tau$.

As in [24], we assume the following characteristics of the scattering responses:

1) $H_{i j}(\tau, t) \neq 0$ only if $(\tau, t) \in \Psi_{R_{i j}} \times \Psi_{T_{i j}}$.

2) $\int\left\|H_{i j}(\tau, t)\right\| d t \neq 0 \forall \tau \in \Psi_{R_{i j}}$.

3) $\int\left\|H_{i j}(\tau, t)\right\| d \tau \neq 0 \forall t \in \Psi_{T_{i j}}$.

4) The point spectrum of $H_{i j}(\cdot, \cdot)$, excluding 0 , is infinite.

5) $H_{i j}(\cdot, \cdot)$ is Lebesgue measurable, that is

$$
\int_{-1}^{1} \int_{-1}^{1}\left|H_{i j}(\tau, t)\right|^{2} d \tau d t<\infty
$$

The first condition means that the scattering response is zero unless the angle of arrival and angle of departure both lie within their respective scattering intervals. The second condition means that in any direction of departure, $t \in \Psi_{T_{i j}}$, there exists at least one path from transmitter $T_{j}$ receiver $R_{i}$. Similarly, the third condition implies that in any direction of arrival, $\tau \in \Psi_{R_{i j}}$, there exists at least one path from $T_{j}$ to $R_{i}$. The fourth condition means that there are many paths from the transmitter to the receiver within the scattering intervals, so that the number of propagation paths that can be resolved within the scattering intervals is limited by the length of the arrays and not by the number of paths. The final condition aids our analysis by ensuring the corresponding integral operator is compact, but is also a physically justified assumption since one could argue for the stricter assumption $\int_{-1}^{1} \int_{-1}^{1}\left|H_{i j}(\tau, t)\right|^{2} d \tau d t \leq 1$, since no more energy can be scattered than is transmitted.

\subsection{Hilbert space of wave-vectors}

We can now write the original input-output relation given in (2) and (3) as

$$
\begin{aligned}
y_{1}(q)= & \int_{\Psi_{R_{11}}} A_{R_{1}}(q, \tau) \int_{\Psi_{T_{11}}} H_{11}(\tau, t) \int_{-L_{T_{1}}}^{L_{T_{1}}} A_{T_{1}}(t, p) x_{1}(p) d \tau d t d p \\
& +\int_{\Psi_{R_{12}}} A_{R_{1}}(q, \tau) \int_{\Psi_{T_{12}}} H_{12}(\tau, t) \int_{-L_{T_{2}}}^{L_{T_{2}}} A_{T_{2}}(t, p) x_{2}(p) d \tau d t d p \\
& +z_{1}(q), \\
y_{2}(q)= & \int_{\Psi_{R_{22}}}^{A_{R_{2}}(q, \tau)} \int_{\Psi_{T_{22}}} H_{22}(\tau, t) \int_{-L_{T_{2}}}^{L_{T_{2}}} A_{T_{2}}(t, p) x_{2}(p) d \tau d t d p \\
& +z_{2}(q) .
\end{aligned}
$$

The channel model of (7) and (8) is expressed in the array domain, that is the transmit and receive signals are expressed as the current distributions excited along the array. Just as one can simplify a signal processing problem by leveraging the Fourier integral to transform from the time domain to the frequency domain, we can leverage the transmit and receive array responses to transform the problem from the array domain to the wave-vector domain. In other words, we can express the transmit and receive signals as field distributions over direction rather 
than current distributions over position along the array. In fact, for our case of the unipolarized linear array, the transmit and receive array responses are the Fourier and inverse-Fourier integral kernels, respectively.

Let $\mathcal{T}_{j}$ be the space of all field distributions that transmitter $T_{j}$ 's array of length $L_{T_{j}}$ can radiate towards the available scattering clusters, $\Psi_{T_{j j}} \cup \Psi_{T_{i j}}$ (both signal-ofinterest and self-interference). In the vernacular of [24], $\mathcal{T}_{j}$ is the space of field distributions array-limited to $L_{T_{j}}$ and wavevector-limited to $\Psi_{T_{i j}} \cup \Psi_{T_{i j}}$. To be precise, define $\mathcal{T}_{j}$ to be the Hilbert space of all square-integrable functions $X_{j}: \Psi_{T_{j j}} \cup \Psi_{T_{i j}} \rightarrow \mathbb{C}$, that can be expressed as

$$
X_{j}(t)=\int_{-L_{T_{j}}}^{L_{T_{j}}} A_{T_{j}}(t, p) x_{j}(p) d p, \quad t \in \Psi_{T_{i j}} \cup \Psi_{T_{i j}}
$$

for some $x_{j}(p), p \in\left[-L_{T_{j}}, L_{T_{j}}\right]$. The inner product defined for this Hilbert Space between two member functions, $U_{j}, V_{j} \in \mathcal{T}_{j}$, is the usual inner product: $\left\langle U_{j}, V_{j}\right\rangle=$ $\int_{\Psi_{T_{i j}} \cup \Psi_{T_{i j}}} U_{j}(t) V_{j}^{*}(t) d t$. Likewise, let $\mathcal{R}_{i}$ be the space of field distributions that can be incident on receiver $R_{i}$ from the available scattering clusters, $\Psi_{R_{i i}} \cup \Psi_{R_{i j}}$, and resolved by an array of length $L_{R_{i}}$. More precisely, $\mathcal{R}_{i}$ is the Hilbert space of all square-integrable functions $Y_{i}: \Psi_{R_{i i}} \cup \Psi_{R_{i j}} \rightarrow$ $\mathbb{C}$, that can be expressed as

$$
Y_{i}(\tau)=\int_{-L_{R_{i}}}^{L_{R_{i}}} A_{R_{i}}^{*}(q, \tau) y_{i}(q) d q, \quad \tau \in \Psi_{R_{i i}} \cup \Psi_{R_{i j}}
$$

for some $y_{i}(q), q \in\left[-L_{R_{i}}, L_{R_{i}}\right]$, with the same inner product. From [24], we know that the dimension of these array-limited and wavevector-limited transmit and receive spaces are, respectively,

$$
\begin{aligned}
\operatorname{dim} \mathcal{T}_{j} & =2 L_{T_{j}}\left|\Psi_{T_{j j}} \cup \Psi_{T_{i j}}\right| \text {, and } \\
\operatorname{dim} \mathcal{R}_{i} & =2 L_{R_{i}}\left|\Psi_{R_{i i}} \cup \Psi_{R_{i j}}\right|
\end{aligned}
$$

We can define the scattering integrals in (7) and (8) as operators mapping from one Hilbert space to another. Define the operator $\mathrm{H}_{i j}: \mathcal{T}_{j} \rightarrow \mathcal{R}_{i}$ by

$$
\left(\mathrm{H}_{i j} X_{j}\right)(\tau)=\int_{\Psi_{T_{i j}} \cup \Psi_{T_{j j}}} H_{i j}(\tau, t) X_{j}(t) d t, \tau \in \Psi_{R_{i j}} \cup \Psi_{R_{i i}} .
$$

We can now write the channel model of (7) and (8) in the wave-vector domain as

$$
\begin{aligned}
& Y_{1}=\mathrm{H}_{11} X_{1}+\mathrm{H}_{12} X_{2}+Z_{1}, \\
& Y_{2}=\mathrm{H}_{22} X_{2}+Z_{2},
\end{aligned}
$$

where $X_{j} \in \mathcal{T}_{j}$, for $j=1,2$ and $Y_{i}, Z_{i} \in \mathcal{R}_{i}$ for $i=1,2$. The following lemma states key properties of the scattering operators in (12-13) that we will leverage in our analysis.

Lemma 1 The scattering operators $\mathrm{H}_{i j},(i, j) \in\{(1,1)$, $(2,2),(1,2)\}$ have the following properties:
1. The scattering operator, $\mathrm{H}_{i j}: \mathcal{T}_{j} \rightarrow \mathcal{R}_{i}$, is a compact operator.

2. The dimension of the range of the scattering operator, $\operatorname{dim} R\left(\mathrm{H}_{i j}\right) \equiv \operatorname{dim} N\left(\mathrm{H}_{i j}\right)^{\perp}$, (i.e., the dimension of the space orthogonal to the operator's nullspace) is given by $\operatorname{dim} R\left(\mathrm{H}_{i j}\right)=2 \min \left\{L_{T_{j}}\left|\Psi_{T_{i j}}\right| L_{R_{i}}\left|\Psi_{R_{i j}}\right|\right\}$.

3. There exists a singular system $\left\{\sigma_{i j}^{(k)}, U_{i j}^{(k)}, V_{i j}^{(k)}\right\}_{k=1}^{\infty}$ for scattering operator $\mathrm{H}_{i j}$, where the singular value $\sigma_{i j}^{(k)}$ is nonzero if and only if $k \leq 2 \min \left\{L_{T_{j}}\left|\Psi_{T_{i j}}\right|, L_{R_{i}}\left|\Psi_{R_{i j}}\right|\right\}$.

Proof Property 1 holds because $H_{i j}(\cdot, \cdot)$, the kernel of integral operator $\mathrm{H}_{i j}$, is square integrable, and an integral operator with a square integrable kernel is compact (see Theorem 8.8 of [32]). Property 2 is just a restatement of the main result of [24]. Property 3 follows from the first two properties: The compactness of $\mathrm{H}_{i j}$, established in Property 1, implies the existence of a singular system, since there exists a singular system for any compact operator (see Section 16.1 of [32]). Property 2 implies that only the first $2 \min \left\{L_{T_{j}}\left|\Psi_{T_{i j}}\right|, L_{R_{i}}\left|\Psi_{R_{i j}}\right|\right\}$ of the singular values will be nonzero, since the $\left\{U_{i j}^{(k)}\right\}$ corresponding to nonzero singular values form a basis for $R\left(\mathrm{H}_{i j}\right)$, which has dimension $2 \min \left\{L_{T_{j}}\left|\Psi_{T_{i j}}\right|, L_{R_{i}}\left|\Psi_{R_{i j}}\right|\right\}$. See Lemma 5 in Appendix $B$ for a description of the properties of singular systems for compact operators, or see Section 2.2 of [33] or Section 16.1 of [32] for a thorough treatment.

\section{Spatial degrees-of-freedom analysis}

We now give the main result of the paper: a characterization of the spatial degrees-of-freedom region for the PBT channel model applied to a full-duplex base station with uplink and downlink flows.

Theorem 1 Let $d_{1}$ and $d_{2}$, respectively, denote the spatial degrees of freedom of the uplink data flow from $T_{1}$ to $R_{1}$, and the downlink data flow from $T_{2}$ to $R_{2}$. The spatial degrees-of-freedom region, $\mathcal{D}_{\mathrm{FD}}$, of the three-node full-duplex channel is the convex hull of all spatial degreesof-freedom tuples, $\left(d_{1}, d_{2}\right)$, satisfying

$$
\begin{aligned}
d_{1} \leq & d_{1}^{\max }=2 \min \left(L_{T_{1}}\left|\Psi_{T_{11}}\right|, L_{R_{1}}\left|\Psi_{R_{11}}\right|\right), \\
d_{2} \leq & d_{2}^{\max }=2 \min \left(L_{T_{2}}\left|\Psi_{T_{22}}\right|, L_{R_{2}}\left|\Psi_{R_{22}}\right|\right), \\
d_{1}+d_{2} \leq & d_{\text {sum }}^{\max }=2 L_{T_{2}}\left|\Psi_{T_{22}} \backslash \Psi_{T_{12}}\right|+2 L_{R_{1}}\left|\Psi_{R_{11}} \backslash \Psi_{R_{12}}\right| \\
& +2 \max \left(L_{T_{2}}\left|\Psi_{T_{12}}\right|, L_{R_{1}}\left|\Psi_{R_{12}}\right|\right) .
\end{aligned}
$$

The degrees-of-freedom region characterized by Theorem $1, \mathcal{D}_{\mathrm{FD}}$, is the pentagon-shaped region shown in Fig. 4. Figure 5a shows a geometric interpretation of the parameters in Theorem 1 . The achievability part of 


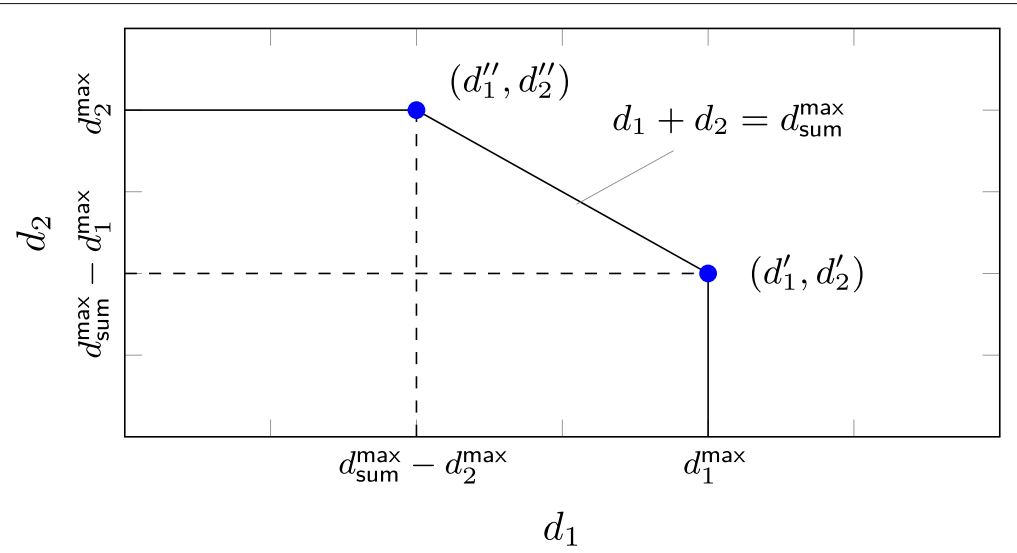

Fig. 4 Degrees-of-freedom region, $\mathcal{D}_{\mathrm{FD}}$

Theorem 1 is given in Section 3.1, and the converse is given in Section 3.2.

\subsection{Achievability}

Overview of achievability proof: Before launching into the full proof, we would like to give a brief sketch of the achievability of corner point $\left(d_{1}^{\prime}, d_{2}^{\prime}\right)$ of the degrees-offreedom region $\mathcal{D}_{\mathrm{FD}}$ shown in Fig. 4 . The steps for achieving the degrees-of-freedom tuple $\left(d_{1}^{\prime}, d_{2}^{\prime}\right)=\left(d_{1}^{\max }, d_{\text {sum }}^{\max }-\right.$ $\left.d_{1}^{\max }\right)$ are illustrated in Fig. 5b.

1. First, the uplink transmitter, $T_{1}$, transmits the maximum number of data streams that the uplink channel will support,

$d_{1}^{\max }=2 \min \left(L_{T_{1}}\left|\Psi_{T_{11}}\right|, L_{R_{1}}\left|\Psi_{R_{11}}\right|\right.$ ) (illustrated by blue arrows in Fig. 5b). The base station downlink transmitter, $T_{2}$, must then structure its transmit signal such that it does not interfere with the base station receiver's reception of these $d_{1}^{\max }$ data streams, as is described in the following steps.

2. Second, the base station transmitter, $T_{2}$, transmits as many data streams as can be supported in the interval $\Psi_{T_{22}} \backslash \Psi_{T_{12}}$ (illustrated by red arrows in Fig. 5b), which is the interval over which signal will couple to the downlink user $R_{2}$, but will not present any self-interference to the base station's own receiver $R_{1}$.

3. Third, the base station transmits as many data streams as possible in the interval $\Psi_{T_{22}} \cap \Psi_{T_{12}}$ while ensuring that the self-interference is only incident on the base station receiver, $R_{1}$ over the interval $\Psi_{R_{11}} \backslash \Psi_{R_{12}}$ (illustrated by green arrows in Fig. 5b), which is the interval over which no uplink signal

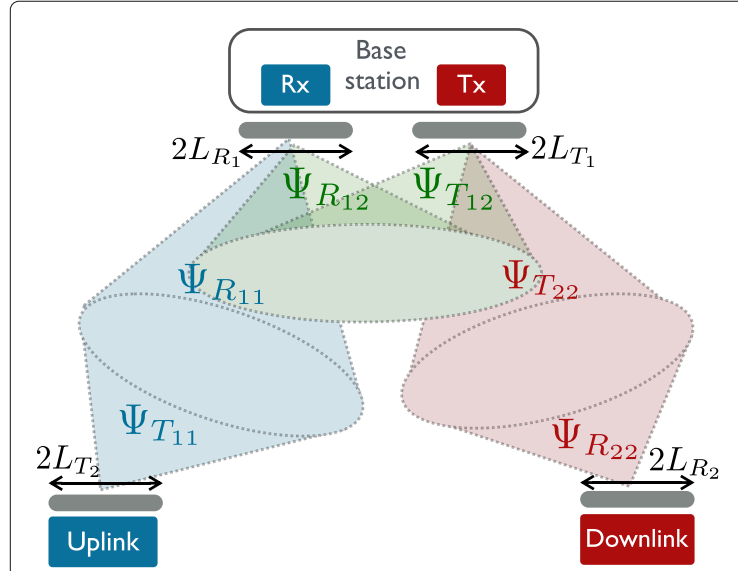

(a) Array lengths and scattering intervals.

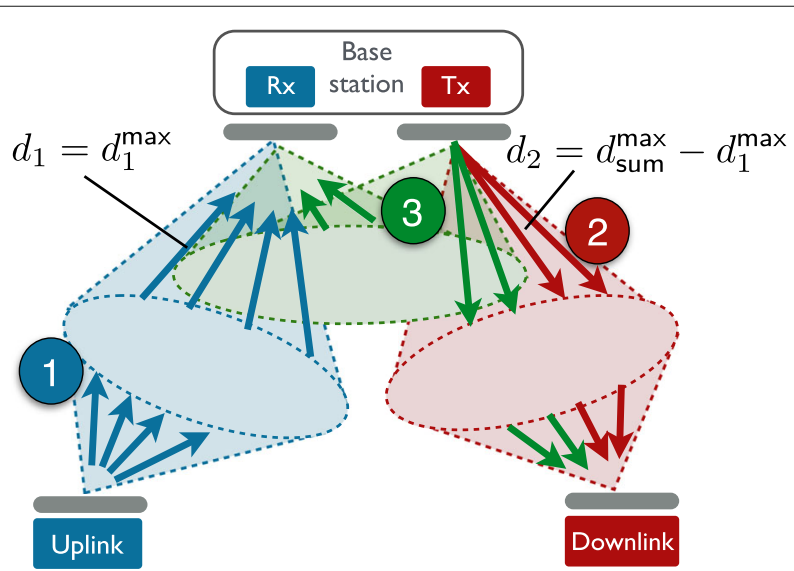

(b) Cartoon illustrating achievability of the corner point $\left(d_{1}, d_{2}\right)=$ $\left(d_{1}^{\max }, d_{\text {sum }}^{\max }-d_{1}^{\max }\right)$

Fig. 5 Diagrams illustrating the geometrical interpretation of the degrees-of-freedom region $\mathcal{D}_{\text {FD }}$ and the achievability strategy. a Array lengths and scattering intervals. b Cartoon illustrating achievability of the corner point $\left(d_{1}, d_{2}\right)=\left(d_{1}^{\max }, d_{\text {sum }}^{\max }-d_{1}^{\max }\right)$ 
form $T_{1}$ will be incident on receiver $R_{1}$. This step occupies a majority of the proof.

The final step in the achievability proof is to show that if the transmission strategies described in steps 1-3 are employed, that the receivers, $R_{1}$ and $R_{2}$, can successfully recover the $d_{1}$ - and $d_{2}$-dimensional data streams, respectively.

Full achievability proof: We establish achievability of $\mathcal{D}_{\mathrm{FD}}$ by way of two lemmas. The first lemma shows the achievability of two specific spatial degrees-of-freedom tuples, and the second shows that these tuples are indeed the corner points of $\mathcal{D}_{\mathrm{FD}}$.

Lemma 2 The spatial degree-of-freedom tuples $\left(d_{1}^{\prime}, d_{2}^{\prime}\right)$ and $\left(d_{1}^{\prime \prime}, d_{2}^{\prime \prime}\right)$, given below, are achievable:

$$
\begin{aligned}
& d_{1}^{\prime}=\min \left\{2 L_{T_{1}}\left|\Psi_{T_{11}}\right|, 2 L_{R_{1}}\left|\Psi_{R_{11}}\right|\right\}, \\
& d_{2}^{\prime}= \begin{cases}\min \left\{d_{T_{2}}, 2 L_{R_{2}}\left|\Psi_{R_{22}}\right|\right\}, & L_{T_{1}}\left|\Psi_{T_{11}}\right| \geq L_{R_{1}}\left|\Psi_{R_{11}}\right|, \\
\min \left\{\delta_{T_{2}}, 2 L_{R_{2}}\left|\Psi_{R_{22}}\right|\right\}, & \text { otherwise }\end{cases} \\
& d_{1}^{\prime \prime}= \begin{cases}\min \left\{2 L_{T_{1}}\left|\Psi_{T_{11}}\right|, d_{R_{1}}\right\}, & L_{R_{2}}\left|\Psi_{R_{22}}\right| \geq L_{T_{2}}\left|\Psi_{T_{22}}\right| \\
\min \left\{2 L_{T_{1}}\left|\Psi_{T_{11}}\right|, \delta_{R_{1}}\right\}, & \text { otherwise }\end{cases}
\end{aligned}
$$

$$
d_{2}^{\prime \prime}=\min \left\{2 L_{T_{2}}\left|\Psi_{T_{22}}\right|, 2 L_{R_{2}}\left|\Psi_{R_{22}}\right|\right\},
$$

where the terms $d_{T_{2}}, \delta_{T_{2}} d_{R_{1}}$, and $\delta_{R_{1}}$ are given in (17-20) within the table at the bottom of the page.

$$
\begin{aligned}
d_{T_{2}}= & 2 L_{T_{2}}\left|\Psi_{T_{22}} \backslash \Psi_{T_{12}}\right| \\
& +2 \min \left\{L_{T_{2}}\left|\Psi_{T_{22}} \cap \Psi_{T_{12}}\right|,\left(L_{T_{2}}\left|\Psi_{T_{12}}\right|-L_{R_{1}}\left|\Psi_{R_{12}}\right|\right)^{+}\right. \\
& \left.+L_{R_{1}}\left|\Psi_{R_{12}} \backslash \Psi_{R_{11}}\right|\right\}
\end{aligned}
$$$$
\delta_{T_{2}}=2 L_{T_{2}}\left|\Psi_{T_{22}} \backslash \Psi_{T_{12}}\right|
$$$$
+2 \min \left\{L_{T_{2}}\left|\Psi_{T_{22}} \cap \Psi_{T_{12}}\right|, L_{T_{2}}\left|\Psi_{T_{12}}\right|\right.
$$$$
-\left[L_{T_{1}}\left|\Psi_{T_{11}}\right|-\left(L_{R_{1}}\left|\Psi_{R_{11}} \backslash \Psi_{R_{12}}\right|\right.\right.
$$$$
\left.\left.\left.+\left(L_{R_{1}}\left|\Psi_{R_{12}}\right|-L_{T_{2}}\left|\Psi_{T_{12}}\right|\right)^{+}\right)\right]\right\}
$$

$$
\begin{aligned}
d_{R_{1}}= & 2 L_{R_{1}}\left|\Psi_{R_{11}} \backslash \Psi_{R_{12}}\right| \\
+ & +2 \min \\
& \left\{L_{R_{1}}\left|\Psi_{R_{11}} \cap \Psi_{R_{12}}\right|,\left(L_{R_{1}}\left|\Psi_{R_{12}}\right|-L_{T_{2}}\left|\Psi_{T_{12}}\right|\right)^{+}\right. \\
& \left.+L_{T_{2}}\left|\Psi_{T_{12}} \backslash \Psi_{T_{22}}\right|\right\}
\end{aligned}
$$

$$
\begin{aligned}
\delta_{R_{1}}= & 2 L_{R_{1}}\left|\Psi_{R_{11}} \backslash \Psi_{R_{12}}\right| \\
+2 \min \{ & \left\{L_{R_{1}}\left|\Omega_{R_{11}} \cap \Psi_{R_{12}}\right|, L_{R_{1}}\left|\Psi_{R_{12}}\right|-\left[L_{R_{2}}\left|\Psi_{R_{22}}\right|\right.\right. \\
& -\left(L_{T_{2}}\left|\Psi_{T_{22}} \backslash \Psi_{T_{12}}\right|+\left(L_{T_{2}}\left|\Psi_{T_{12}}\right|\right.\right. \\
& \left.\left.\left.\left.-L_{R_{1}}\left|\Psi_{R_{12}}\right|\right)^{+}\right)\right]\right\}
\end{aligned}
$$

Proof Due to the symmetry of the problem, it suffices to demonstrate achievability of only the first spatial degreeof-freedom pair in Lemma $2,\left(d_{1}^{\prime}, d_{2}^{\prime}\right)$, as the second pair, $\left(d_{1}^{\prime \prime}, d_{2}^{\prime \prime}\right)$, follows from symmetry. Thus we seek to prove the achievability of the tuple $\left(d_{1}^{\prime}, d_{2}^{\prime}\right)$ given in (17-18). We will show achievability of $\left(d_{1}^{\prime}, d_{2}^{\prime}\right)$ in the case where $L_{T_{1}}\left|\Psi_{T_{11}}\right| \geq L_{R_{1}}\left|\Psi_{R_{11}}\right|$, for which

$$
\begin{aligned}
& d_{1}^{\prime}=2 L_{R_{1}}\left|\Psi_{R_{11}}\right|, \\
& d_{2}^{\prime}=\min \left\{d_{T_{2}}, 2 L_{R_{2}}\left|\Psi_{R_{22}}\right|\right\},
\end{aligned}
$$

where

$$
\begin{aligned}
d_{T_{2}}= & 2 L_{T_{2}}\left|\Psi_{T_{22}} \backslash \Psi_{T_{12}}\right| \\
& +\min \left\{\begin{array}{c}
2 L_{T_{2}}\left|\Psi_{T_{22}} \cap \Psi_{T_{12}}\right|, \\
2\left(L_{T_{2}}\left|\Psi_{T_{12}}\right|-L_{R_{1}}\left|\Psi_{R_{12}}\right|\right)^{+}+2 L_{R_{1}}\left|\Psi_{R_{12}} \backslash \Psi_{R_{11}}\right|
\end{array}\right\} .
\end{aligned}
$$

Achievability of $\left(d_{1}^{\prime}, d_{2}^{\prime}\right)$ in the $L_{T_{1}}\left|\Psi_{T_{11}}\right|<L_{R_{1}}\left|\Psi_{R_{11}}\right|$ case is analogous.

We now begin the steps to show achievability of (25) and (26).

\subsubsection{Defining key subspaces}

We first define key subspaces of the transmit and receive wave-vector spaces $\left(\mathcal{T}_{1}, \mathcal{T}_{2}, \mathcal{R}_{1}\right.$, and $\left.\mathcal{R}_{2}\right)$ that will be crucial in demonstrating achievability.

Subspaces of $\mathcal{T}_{2}$ : Recall that $\mathcal{T}_{2}$ is the space of all field distributions that can be radiated by the base station transmitter, $T_{2}$, in the direction of the scatterer intervals, $\Psi_{T_{22}} \cup \Psi_{T_{12}}$, (both signal-of-interest and self-interference). Let $\mathcal{T}_{22 \backslash 12} \subseteq \mathcal{T}_{2}$ be the subspace of field distributions that can be transmitted by $T_{2}$, which are nonzero only in the interval $\Psi_{T_{22}} \backslash \Psi_{T_{12}}$,

$$
\mathcal{T}_{22 \backslash 12} \equiv \operatorname{span}\left\{X_{2} \in \mathcal{T}_{2}: X_{2}(t)=0 \forall t \in \Psi_{T_{12}}\right\} .
$$

More intuitively, $\mathcal{T}_{22 \backslash 12}$ is the space of transmissions from the base station which couple only to the intended downlink user, and do not couple back to the base station receiver as self-interference. Similarly let $\mathcal{T}_{12} \subseteq \mathcal{T}_{2}$ be the subspace of functions that are only nonzero in the interval $\Psi_{T_{12}}$,

$$
\mathcal{T}_{12} \equiv \operatorname{span}\left\{X_{2} \in \mathcal{T}_{2}: X_{2}(t)=0 \forall t \notin \Psi_{T_{12}}\right\},
$$

that is, the space of base station transmissions which $d o$ couple to the base station receiver as self-interference. Finally, let $\mathcal{T}_{22 \cap 12} \subseteq \mathcal{T}_{12} \subseteq \mathcal{T}_{2}$ be the subspace of field distributions that are nonzero only in the interval $\Psi_{T_{22}} \cap$ $\Psi_{T_{12}}$,

$$
\mathcal{T}_{22 \cap 12} \equiv \operatorname{span}\left\{X_{2} \in \mathcal{T}_{2}: X_{2}(t)=0 \forall t \notin \Psi_{T_{22}} \cap \Psi_{T_{12}}\right\},
$$

the space of base station transmission which couple both to the downlink user and to the base station receiver. From 
the result of [24], we know that the dimension of each of these transmit subspaces of $\mathcal{T}_{1}$ is as follows:

$$
\begin{aligned}
\operatorname{dim} \mathcal{T}_{12} & =2 L_{T_{2}}\left|\Psi_{T_{12}}\right| \\
\operatorname{dim} \mathcal{T}_{22 \backslash 12} & =2 L_{T_{2}}\left|\Psi_{T_{22}} \backslash \Psi_{T_{12}}\right|, \\
\operatorname{dim} \mathcal{T}_{22 \cap 12} & =2 L_{T_{2}}\left|\Psi_{T_{22}} \cap \Psi_{T_{12}}\right| .
\end{aligned}
$$

Definition 1 We say that Hilbert space $\mathcal{A}$ is the orthogonal direct sum of Hilbert spaces $\mathcal{B}$ and $\mathcal{C}$ if any $a \in \mathcal{A}$ can be decomposed as $a=b+c$, for some $b \in \mathcal{B}$ and $c \in \mathcal{C}$, where $a$ and $b$ are orthogonal. We use the notation $\mathcal{A}=\mathcal{B} \oplus \mathcal{C}$ to denote that $A$ is the orthogonal direct sum of $\mathcal{B}$ and $\mathcal{C}$.

One can check that $\mathcal{T}_{12}$ and $\mathcal{T}_{22 \backslash 12}$ are constructed such that they form an orthogonal direct sum for space $\mathcal{T}_{2}$ :

$$
\mathcal{T}_{2}=\mathcal{T}_{12} \oplus \mathcal{T}_{22 \backslash 12},
$$

thus any $X_{2} \in \mathcal{T}_{2}$ can be written as $X_{2}=X_{2_{\text {orth }}}+X_{2 \text { lnt }}$, for some $X_{2 \text { orth }} \in \mathcal{T}_{22 \backslash 12}$ and $X_{2 \text { Int }} \in \mathcal{T}_{12}$, such that $X_{2 \text { orth }} \perp$ $X_{2 \text { |nt }}$. By the construction of $\mathcal{T}_{22 \backslash 12}, \mathrm{H}_{12} X_{2 \text { orth }}=0$, since $H_{12}(\tau, t)=0 \forall t \notin \Psi_{T_{12}}$ and $X_{2_{\text {orth }}} \in \mathcal{T}_{22 \backslash 12}$ implies $X_{2_{\text {orth }}}(t)=0 \forall t \in \Psi_{T_{12}}$. In other words, $X_{2_{\text {orth }}} \in \mathcal{T}_{22 \backslash 12}$ is zero everywhere the integral kernel $H_{12}(\tau, t)$ is nonzero. Thus, any transmitted field distribution that lies in the subspace $\mathcal{T}_{22 \backslash 12}$ will not present any interference to $R_{2}$.

Subspaces of $\mathcal{T}_{1}$ : Recall that $\mathcal{T}_{1}$ is the space of all field distributions that can be radiated by the uplink user transmitter, $T_{1}$, towards the available scatterers. Let $\mathcal{T}_{11} \subseteq \mathcal{T}_{1}$ be the subspace of field distributions that can be transmitted by $T_{1}$ 's continuous linear array of length $L_{T_{1}}$ which are nonzero only in the interval $\Psi_{T_{11}}$, more precisely

$$
\mathcal{T}_{11} \equiv \operatorname{span}\left\{X_{1} \in \mathcal{T}_{1}: X_{1}(t)=0 \forall t \notin \Psi_{T_{11}}\right\}
$$

More intuitively, $\mathcal{T}_{11}$ is the space of transmissions from the uplink user which will couple to the base station receiver. From the result of [24], we know that

$$
\operatorname{dim} \mathcal{T}_{11}=2 L_{T_{1}}\left|\Psi_{T_{11}}\right| .
$$

Note that $\mathcal{T}_{11}=\mathcal{T}_{1}$, since we have assumed $\Psi_{T_{21}}=\emptyset$. Although $\mathcal{T}_{11}$ is thus redundant, we define it for notational consistency.

Subspaces of $\mathcal{R}_{1}$ : Recall that $\mathcal{R}_{1}$ is the space of all incident field distributions that can be resolved by the base station receiver, $R_{1}$. Let $\mathcal{R}_{12} \subseteq \mathcal{R}_{1}$ to be the subspace of received field distributions which are nonzero only for $\tau \in \Psi_{R_{12}}$, that is

$$
\mathcal{R}_{12} \equiv \operatorname{span}\left\{Y_{1} \in \mathcal{R}_{1}: Y_{1}(\tau)=0 \forall \tau \notin \Psi_{R_{12}}\right\} .
$$

Less formally, $\mathcal{R}_{12}$ is the space of receptions at the base station which could have emanated from the base stations own transmitter. Similarly, let $\mathcal{R}_{12 \backslash 11} \subseteq \mathcal{R}_{12} \subseteq \mathcal{R}_{1}$ be the subspace of received field distributions that are only nonzero for $\tau \in \Psi_{R_{12}} \backslash \Psi_{R_{11}}$,

$$
\mathcal{R}_{12 \backslash 11} \equiv \operatorname{span}\left\{Y_{1} \in \mathcal{R}_{1}: Y_{1}(\tau)=0 \forall \tau \in \Psi_{R_{11}}\right\} .
$$

Less formally, $\mathcal{R}_{12 \backslash 11}$ is the space of receptions at the base station which could have emanated from the base station transmitter, but could not have emanated from the uplink user. Finally, define $\mathcal{R}_{11} \subseteq \mathcal{R}_{1}$ to be the subspace of received field distributions that are nonzero only for $\tau \in \Psi_{R_{11}}$,

$$
\mathcal{R}_{11} \equiv \operatorname{span}\left\{Y_{1} \in \mathcal{R}_{1}: Y_{1}(\tau)=0 \forall \tau \notin \Psi_{R_{11}}\right\},
$$

the space of base station receptions which could have emanated from the intended uplink user. Note that $\mathcal{R}_{1}=$ $\mathcal{R}_{11} \oplus \mathcal{R}_{12 \backslash 11}$. From the result of [24], we know the dimension of each of the above base-station receive subspaces is as follows:

$$
\begin{aligned}
\operatorname{dim} \mathcal{R}_{11} & =2 L_{R_{1}}\left|\Psi_{R_{11}}\right|, \\
\operatorname{dim} \mathcal{R}_{12 \backslash 11} & =2 L_{R_{1}}\left|\Psi_{R_{12}} \backslash \Psi_{R_{11}}\right|, \\
\operatorname{dim} \mathcal{R}_{12} & =2 L_{R_{1}}\left|\Psi_{R_{12}}\right| .
\end{aligned}
$$

Subspaces of $\mathcal{R}_{2}$ : Recall that $\mathcal{R}_{2}$ is the space of all incident field distributions that can be resolved by the downlink user receiver, $R_{2}$. Let $\mathcal{R}_{22} \subseteq \mathcal{R}_{2}$ to be the subspace of received field distributions which are nonzero only for $\tau \in \Psi_{R_{22}}$, that is

$$
\mathcal{R}_{22} \equiv \operatorname{span}\left\{Y_{2} \in \mathcal{R}_{2}: Y_{2}(\tau)=0 \forall \tau \notin \Psi_{R_{22}}\right\} .
$$

Note that $\mathcal{R}_{22}=\mathcal{R}_{2}$, since we have assumed $\Psi_{R_{21}}=\emptyset$. Although $\mathcal{R}_{22}$ is thus redundant, we define it for notational consistency. By substituting the subspace dimensions given above into (25) and (27), we can restate the degree-of-freedom pair whose achievability we are establishing as

$$
\left(d_{1}^{\prime}, d_{2}^{\prime}\right)=\left(\operatorname{dim} \mathcal{R}_{11}, \min \left\{d_{T_{2}}, \operatorname{dim} \mathcal{R}_{22}\right\}\right), \text { where }
$$

$$
\begin{aligned}
d_{T_{2}}= & \operatorname{dim} \mathcal{T}_{22 \backslash 12} \\
& +\min \left\{\begin{array}{c}
\operatorname{dim} \mathcal{T}_{22 \cap 12} \\
\left(\operatorname{dim} \mathcal{T}_{12}-\operatorname{dim} \mathcal{R}_{12}\right)^{+}+\operatorname{dim} \mathcal{R}_{12 \backslash 11}
\end{array}\right\}
\end{aligned}
$$

Now that we have defined the relevant subspaces, we can show how these subspaces are leveraged in the transmission and reception scheme that achieves the spatial degrees-of-freedom tuple $\left(d_{1}^{\prime}, d_{2}^{\prime}\right)$.

\subsubsection{Spatial processing at each transmitter/receiver}

We now give the transmission schemes at each transmitter and the recovery schemes at each receiver. 
Processing at uplink user transmitter, $T_{1}$ : Recall that $d_{1}^{\prime}=\operatorname{dim} \mathcal{R}_{11}$ is the number of spatial degrees-offreedom we wish to achieve for Flow ${ }_{1}$, the uplink flow. Let $\left\{\chi_{1}^{(k)}\right\}_{k=1}^{d_{1}^{\prime}}, \chi_{1}^{(i)} \in \mathbb{C}$, be the $d_{1}^{\prime}$ symbols that $T_{1}$ wishes to transmit to $R_{1}$. We know from Lemma 1 that there exists a singular value expansion for $\mathrm{H}_{11}$, so let $\left\{\sigma_{11}^{(k)}, U_{11}^{(k)}, V_{11}^{(k)}\right\}_{k=1}^{\infty}$ be a singular system for the operator $\mathrm{H}_{11}: \mathcal{T}_{1} \rightarrow \mathcal{R}_{1}$ (see Lemma 5 in Appendix B for the definition of a singular system).

Note that the functions $\left\{V_{11}^{(k)}\right\}_{k=1}^{\operatorname{dim} \mathcal{T}_{1}}$ form an orthonormal basis for $\mathcal{T}_{1}$, and since $d_{1}^{\prime}=\operatorname{dim} \mathcal{R}_{11} \leq \operatorname{dim} \mathcal{T}_{1}$, there are at least as many such basis functions as there are symbols to transmit.

We construct $X_{1}$, the transmit wave-vector signal transmitted by $T_{1}$, as

$$
X_{1}=\sum_{k=1}^{d_{1}^{\prime}} \chi_{1}^{(k)} V_{11}^{(k)}
$$

\section{Processing at the base station transmitter, $T_{2}$ :}

Recall that $d_{2}^{\prime}=\min \left\{d_{T_{2}}, 2 L_{R_{2}}\left|\Psi_{R_{22}}\right|\right\}$, where $d_{T_{2}}$ is given in (27), is the number of spatial degrees-of-freedom we wish to achieve for Flow 2 , the downlink flow. Let $\left\{\chi_{2}^{(k)}\right\}_{k=1}^{d_{2}^{\prime}}$ be the $d_{2}^{\prime}$ symbols that $T_{2}$ wishes to transmit to $R_{2}$. We split the $T_{2}$ transmit signal into the sum of two orthogonal components, $X_{2 \text { orth }} \in \mathcal{T}_{22 \backslash 12}$ and $X_{2 \text { |nt }} \in \mathcal{T}_{12}$, so that the wave-vector signal transmitted by $T_{2}$ is

$$
X_{2}=X_{2_{\text {orth }}}+X_{2 \text { lnt }}, \quad X_{2 \text { orth }} \in \mathcal{T}_{22 \backslash 12}, \quad X_{2 \text { lnt }} \in \mathcal{T}_{12} .
$$

Recall that $X_{2 \text { orth }} \in \mathcal{T}_{22 \backslash 12}$ implies $\mathrm{H}_{12} X_{2_{\text {Orth }}}=0$. Thus, we can construct $X_{2_{\text {orth }}} \in \mathcal{T}_{22 \backslash 12}$ without regard to the structure of $\mathrm{H}_{12}$. Let $\left\{Q_{22 \backslash 12}^{(i)}\right\}_{i=1}^{\operatorname{dim} \mathcal{T}_{22 \backslash 12}}$ be an arbitrary orthonormal basis for $\mathcal{T}_{22 \backslash 12}$, and let

$$
d_{2_{\text {Orth }}}^{\prime} \equiv \min \left\{\operatorname{dim} \mathcal{T}_{22 \backslash 12}, \operatorname{dim} \mathcal{R}_{22}\right\},
$$

be the number of symbols that $T_{2}$ will transmit along $X_{2_{\text {orth }}}$. We construct $X_{2_{\text {orth }}}$ as

$$
X_{2 \mathrm{Orth}}=\sum_{i=1}^{d_{2 \mathrm{Orth}}^{\prime}} \chi_{2}^{(i)} Q_{22 \backslash 12}^{(i)}
$$

Recall that there are $d_{2}^{\prime}$ total symbols that $T_{2}$ wishes to transmit, and we have transmitted $d_{2 \text { orth }}^{\prime}$ symbols along $X_{2_{\text {Orth }}}$, thus there are $d_{2}^{\prime}-d_{2_{\text {Orth }}}^{\prime}$ symbols remaining to transmit along $X_{2 \text { Int }}$. Let

$$
\begin{aligned}
d_{2 \text { lnt }}^{\prime} & \equiv d_{2}^{\prime}-d_{2_{\text {Orth }}^{\prime}}^{\prime} \\
& =\min \left\{\begin{array}{c}
\operatorname{dim} \mathcal{T}_{22 \cap 12} \\
\left(\operatorname{dim} \mathcal{T}_{12}-\operatorname{dim} \mathcal{R}_{12}\right)^{+}+\operatorname{dim} \mathcal{R}_{12 \backslash 11}, \\
\left(\operatorname{dim} \mathcal{R}_{22}-\operatorname{dim} \mathcal{T}_{22 \backslash 12}\right)^{+}
\end{array}\right\} .
\end{aligned}
$$

Now since $X_{2 \text { lnt }} \in \mathcal{T}_{12}, \mathrm{H}_{12} X_{2 \text { lnt }}$ is nonzero in general, $X_{2 \text { |nt }}$ will present interference to $R_{1}$. Therefore, we must construct $X_{2 \text { lnt }}$ such that it communicates $d_{2 \text { lnt }}^{\prime}$ symbols to $R_{2}$, without impeding $R_{1}$ from recovering the $d_{1}^{\prime}$ symbols transmitted from $T_{1}$. Thus, the construction of $X_{2 \text { nnt }} \in \mathcal{T}_{12}$ will indeed depend on the structure of $\mathrm{H}_{12}$.

First consider the case where $\operatorname{dim} \mathcal{T}_{12} \leq \operatorname{dim} \mathcal{R}_{12}$. In this case, Eq. (50), which gives the number of symbols that must be transmitted along $X_{2 \text { |nt }}$, simplifies to $d_{2 \text { nnt }}^{\prime}=$ $\min \left\{\operatorname{dim} \mathcal{T}_{22 \cap 12}, \operatorname{dim} \mathcal{R}_{12 \backslash 11},\left(\operatorname{dim} \mathcal{R}_{22}-\operatorname{dim} \mathcal{T}_{22 \backslash 12}\right)^{+}\right\}$.

Let $\left\{\sigma_{12}^{(k)}, U_{12}^{(k)}, V_{12}^{(k)}\right\}_{k=1}^{\infty}$ be a singular system for $\mathrm{H}_{12}$. From Property 3 of Lemma 1 , we know that $\sigma_{12}^{(k)}$ is zero for $k>\operatorname{dim} \mathcal{T}_{12}$ and nonzero for $k \leq \operatorname{dim} \mathcal{T}_{12}$. Note that $\left\{V_{12}^{(k)}\right\}_{k=1}^{\operatorname{dim} \mathcal{T}_{12}}$ is an orthonormal basis for $\mathcal{T}_{12}$. In the case of $\operatorname{dim} \mathcal{T}_{12} \leq \operatorname{dim} \mathcal{R}_{12}$ (the case for which are constructing $X_{2 \operatorname{lnt}}$ ), we have

$$
\begin{aligned}
d_{2 \text { nnt }}^{\prime}= & \min \left\{\operatorname{dim} \mathcal{T}_{22 \cap 12}, \operatorname{dim} \mathcal{R}_{12 \backslash 11},\left(\operatorname{dim} \mathcal{R}_{22}\right.\right. \\
& \left.\left.-\operatorname{dim} \mathcal{T}_{22 \backslash 12}\right)^{+}\right\} \\
\leq & \operatorname{dim} \mathcal{T}_{22 \cap 12} \leq \operatorname{dim} \mathcal{T}_{12}
\end{aligned}
$$

so that there are at least as many $V_{12}^{(k)}$ 's as there are symbols to transmit along $X_{2 \text { lnt }}$. We construct $X_{2 \text { int }}$ as

$$
X_{2 \operatorname{lnt}}=\sum_{k=1}^{d_{2 \operatorname{lnt}}^{\prime}} \chi_{2}^{\left(k+d_{2 \text { Orth }}^{\prime}\right)} V_{12}^{(k)} \text {. }
$$

Now we will consider the construction of $X_{2 \operatorname{lnt}}$ for the other case where $\operatorname{dim} \mathcal{T}_{12}>\operatorname{dim} \mathcal{R}_{12}$. In the $\operatorname{dim} \mathcal{T}_{12}>$ $\operatorname{dim} \mathcal{R}_{12}$ case Eq. (50), which gives the number of symbols that must be transmitted along $X_{2 \text { |nt }}$, simplifies to

$$
d_{2 \text { Int }}^{\prime}=\min \left\{\begin{array}{c}
\operatorname{dim} \mathcal{T}_{22 \cap 12}, \\
\left(\operatorname{dim} \mathcal{T}_{12}-\operatorname{dim} \mathcal{R}_{12}\right)+\operatorname{dim} \mathcal{R}_{12 \backslash 11}, \\
\left(\operatorname{dim} \mathcal{R}_{22}-\operatorname{dim} \mathcal{T}_{22 \backslash 12}\right)^{+}
\end{array}\right\} .
$$

Note that the signal that $R_{1}$ receives from $T_{1}$ will lie only in $\mathcal{R}_{11}$. Thus, if we can ensure that the signal from $T_{2}$ falls in the orthogonal space, $\mathcal{R}_{12 \backslash 11}$, then we have avoided interference. Let $\mathrm{H}_{12}^{\prime \prime}: \mathcal{T}_{12} \rightarrow \mathcal{R}_{12}$ be the restriction of $\mathrm{H}_{12}: \mathcal{T}_{2} \rightarrow \mathcal{R}_{1}$ to domain $\mathcal{T}_{12}$ and codomain $\mathcal{R}_{12}$. We consider the constriction, $\mathrm{H}_{12}^{\prime \prime}$, instead of $\mathrm{H}_{12}$ so that the preimage under $\mathrm{H}_{12}^{\prime \prime}$ is a subset of $\mathcal{T}_{12}$, so that any functions within this preimage have not already been used in constructing $X_{2_{\text {orth }}}$. We can characterize the requirement that $Y_{1}(\tau)$ 
not be interfered over $\tau \in \Psi_{R_{11}}$ as $\mathrm{H}_{12}^{\prime \prime} X_{2 \text { |nt }} \in \mathcal{R}_{12 \backslash 11}$, or equivalently $X_{2 \text { lnt }} \in \mathcal{P}_{12 \backslash 11}$, where

$$
\mathcal{P}_{12 \backslash 11} \equiv \mathrm{H}_{12}^{\prime \prime} \leftarrow\left(\mathcal{R}_{12 \backslash 11}\right) \subseteq \mathcal{T}_{12}
$$

is the preimage of $\mathcal{R}_{12 \backslash 11}$ under $\mathrm{H}_{12}^{\prime \prime}$. Thus, any function in $\mathcal{P}_{12 \backslash 11}$ can be used for signaling to $R_{2}$ without interfering $X_{1}$ at $R_{1}$. The number of symbols that can be transmitted will thus depend on the dimension of this interferencefree preimage. Corollary 1 in the appendix states that if $\mathrm{C}: \mathcal{X} \rightarrow \mathcal{Y}$ is a linear operator with closed range, and $\mathcal{S}$ is a subspace of the range of $C, \mathcal{S} \subset R(C)$, then $\operatorname{dim} C^{\leftarrow}(\mathcal{S})=$ $\operatorname{dim} N(\mathrm{C})+\operatorname{dim}(\mathcal{S})$. Note that $R\left(\mathrm{H}_{12}^{\prime \prime}\right)$ has finite dimension (namely $2 \min \left\{L_{T_{2}} \Psi_{T_{12}}, L_{R_{1}} \Psi_{R_{12}}\right\}<\infty$ ), and since any finite dimensional subspace of a normed space is closed, $R\left(\mathrm{H}_{12}^{\prime \prime}\right)$ is closed. Further, note that since we are considering the case where $\operatorname{dim} \mathcal{T}_{12}>\operatorname{dim} \mathcal{R}_{12}$, it is easy to see that $R\left(\mathrm{H}_{12}^{\prime \prime}\right)=\mathcal{R}_{12}$, which implies $\mathcal{R}_{12 \backslash 11} \subseteq R\left(\mathrm{H}_{12}^{\prime \prime}\right)$, since $\mathcal{R}_{12 \backslash 11} \subseteq \mathcal{R}_{12}$ by construction. Thus, the linear operator $\mathrm{H}_{12}^{\prime \prime}: \mathcal{T}_{12} \rightarrow \mathcal{R}_{12}$ and the subspace $\mathcal{R}_{12 \backslash 11}$ satisfy the conditions on operator $C$ and subspace $\mathcal{S}$, respectively, in the hypothesis of Corollary 1 . Thus, we can apply Corollary 1 to show that, when $\operatorname{dim} \mathcal{T}_{12}>\operatorname{dim} \mathcal{R}_{12}$, the dimension of $\mathcal{P}_{12 \backslash 11}$ is given by

$$
\begin{aligned}
\operatorname{dim} \mathcal{P}_{12 \backslash 11} & =\operatorname{dim} N\left(\mathrm{H}_{12}^{\prime \prime}\right)+\operatorname{dim} \mathcal{R}_{1 \backslash 11} \\
& =\left(\operatorname{dim} \mathcal{T}_{12}-\operatorname{dim} \mathcal{R}_{12}\right)+\operatorname{dim} \mathcal{R}_{12 \backslash 11} \\
& \geq \min \left\{\begin{array}{c}
\operatorname{dim} \mathcal{T}_{22 \cap 12} \\
\left(\operatorname{dim} \mathcal{T}_{12}-\operatorname{dim} \mathcal{R}_{12}\right)+\operatorname{dim} \mathcal{R}_{12 \backslash 11} \\
\left(\operatorname{dim} \mathcal{R}_{22}-\operatorname{dim} \mathcal{T}_{22 \backslash 12}\right)^{+}
\end{array}\right\} \\
& =d_{2_{\text {|nt }}^{\prime}}^{\prime}, \quad \operatorname{dim} \mathcal{T}_{12}>\operatorname{dim} \mathcal{R}_{12} .
\end{aligned}
$$

Therefore, the dimension of $\mathcal{P}_{12 \backslash 11}$, the preimage of $\mathcal{R}_{12 \backslash 11}$ under $\mathrm{H}_{12}^{\prime \prime}$, is indeed large enough to allow $T_{2}$ to transmit the remaining $d_{2 \text { Int }}^{\prime}$ symbols along the basis functions of $\operatorname{dim} \mathcal{P}_{12 \backslash 11}$. Let $\left\{P_{12}^{(i)}\right\}_{i=1}^{\operatorname{dim} \mathcal{P}_{12 \backslash 11}}$ be an orthonormal basis for $\mathcal{P}_{12 \backslash 11}$. Then, we construct $X_{2 \text { |nt }}$ as

$$
X_{2 \mathrm{lnt}}=\sum_{k=1}^{d_{2 \operatorname{lnt}}^{\prime}} \chi_{2}^{\left(k+d_{2 \mathrm{Orhh}}^{\prime}\right)} P_{12}^{(k)}
$$

In summary, combining all cases we see that the wavevector transmitted by $T_{2}$ is

$$
\begin{aligned}
X_{2}= & X_{2_{\text {Orth }}}+X_{2 \text { Int }}=\sum_{i=1}^{d_{2_{\text {Orth }}}^{\prime}} \chi_{2}^{(i)} Q_{22 \backslash 12}^{(i)}+\sum_{k=1}^{d_{2 \text { Int }}^{\prime}} \chi_{2}^{\left(k+d_{2_{\text {Orth }}^{\prime}}\right)} \\
& \times\left(V_{12}^{(k)} 1\left(\operatorname{dim} \mathcal{T}_{12} \leq \operatorname{dim} \mathcal{R}_{12}\right)+P_{12}^{(k)} 1\left(\operatorname{dim} \mathcal{T}_{12}>\operatorname{dim} \mathcal{R}_{12}\right)\right)
\end{aligned}
$$

$$
\begin{array}{r}
=\sum_{i=1}^{d_{2 \text { orth }}^{\prime \prime}} \chi_{2}^{(i)} Q_{22 \backslash 12}^{(i)}+\sum_{i=1+d_{2 \text { orh }}^{\prime}}^{d_{2}^{\prime}} \chi_{2}^{(i)}\left(V_{12}^{\left(i-d_{2 \text { Orth }}^{\prime \prime}\right)} 1\left(\operatorname{dim} \mathcal{T}_{12} \leq \operatorname{dim} \mathcal{R}_{12}\right)\right. \\
\left.+P_{12}^{\left(i-d_{2 \text { Orth }}^{\prime}\right)} 1\left(\operatorname{dim} \mathcal{T}_{12}>\operatorname{dim} \mathcal{R}_{12}\right)\right)
\end{array}
$$

$$
\begin{aligned}
& =\sum_{i=1}^{d_{2}^{\prime}} \chi_{2}^{(i)} B_{2}^{(i)} \text {, where } \\
& B_{2}^{(i)}=\left\{\begin{array}{lr}
Q_{22 \backslash 12}^{(i)} & : i \leq d_{2 \mathrm{Orth}}^{\prime} \\
V_{12}^{\left(i-d_{2 \mathrm{Orth}}^{\prime}\right)}: i>d_{2_{\mathrm{Orth}}}^{\prime}, \operatorname{dim} \mathcal{T}_{12} \leq \operatorname{dim} \mathcal{R}_{12} . \\
P_{12}^{\left(i-d_{2 \mathrm{Orth}}^{\prime}\right)}: i>d_{2_{\mathrm{Orth}}}^{\prime}, \operatorname{dim} \mathcal{T}_{12}>\operatorname{dim} \mathcal{R}_{12}
\end{array}\right.
\end{aligned}
$$

Now that we have constructed $X_{1}$, the uplink wavevector signal transmitted on the the uplink user, and $X_{2}$, the wavevector signal transmitted on the dowlink by the base station, we show how the base station receiver, $R_{1}$ and the downlink user $R_{2}$ process their received signals to detect the original information-bearing symbols.

Processing at the base station receiver, $R_{1}$ : We need to show that $R_{1}$ can obtain at least $d_{1}^{\prime}=\operatorname{dim} \mathcal{R}_{11}$ independent linear combinations of the $d_{1}^{\prime}$ symbols transmitted from $T_{1}$, and that each of these linear combinations are corrupted only by noise, and not interference from $T_{2}$.

In the case where $\operatorname{dim} \mathcal{T}_{12}>\operatorname{dim} \mathcal{R}_{12}, T_{2}$ constructed $X_{2}$ such that $\mathrm{H}_{12} X_{2}$ is orthogonal to any function in $\mathcal{R}_{11}$. Therefore, $R_{1}$ can eliminate interference from $T_{2}$ by simply projecting $Y_{1}$ onto $\mathcal{R}_{11}$ to recover the $\operatorname{dim} \mathcal{R}_{11}$ linear combinations it needs. We now formalize this projection onto $\mathcal{R}_{11}$. Recall that the set of left-singular functions of $\mathrm{H}_{11},\left\{U_{11}^{(l)}\right\}_{l=1}^{\operatorname{dim} \mathcal{R}_{11}}$, form an orthonormal basis for $\mathcal{R}_{11}$. In the case where $\operatorname{dim} \mathcal{T}_{12}>\operatorname{dim} \mathcal{R}_{12}$, receiver $R_{2}$ constructs the set of complex scalars $\left\{\xi_{1}^{(l)}\right\}_{l=1}^{\operatorname{dim} \mathcal{R}_{11}}, \quad \xi_{1}^{(l)}=$ $\left\langle Y_{1}, U_{11}^{(l)}\right\rangle$. One can check that result of each of these projections is

$$
\xi_{1}^{(l)}=\sigma_{11}^{(l)} \chi_{1}^{(l)}+\left\langle Z_{1}, U_{11}^{(l)}\right\rangle, \quad l=1,2, \ldots, \operatorname{dim} \mathcal{R}_{11},
$$

and thus obtains each of the $d_{1}^{\prime}=\operatorname{dim} \mathcal{R}_{11}$ linear combinations of the intended symbols corrupted only by noise, as desired. Moreover, in this case the obtained linear combinations are already diagonalized, with the $l$ th projection only containing a contribution from the $l$ th desired symbol.

In the case where $\operatorname{dim} \mathcal{T}_{12} \leq \operatorname{dim} \mathcal{R}_{12}, \mathrm{H}_{12} X_{2}$ in general will not be orthogonal to every function in $\mathcal{R}_{11}$, and some slightly more sophisticated processing must be performed to decouple the interference from the signal of interest. 
First, $R_{1}$ can recover $\operatorname{dim} \mathcal{R}_{11 \backslash 12}$ interference-free linear combinations by projecting its received signal, $Y_{1}$, onto $\mathcal{R}_{11 \backslash 12}$. Let $\left\{J_{11 \backslash 12}^{(l)}\right\}_{l=1}^{\operatorname{dim} \mathcal{R}_{11 \backslash 12}}$ be an orthonormal basis for $\mathcal{R}_{11 \backslash 12}$. Receiver $R_{1}$ forms a set of complex scalars

$$
\left\{\xi_{1}^{(l)}\right\}_{l=1}^{\operatorname{dim} \mathcal{R}_{11 \backslash 12}}, \quad \xi_{1}^{(l)}=\left\langle Y_{1}, J_{11 \backslash 12}^{(l)}\right\rangle
$$

Note that each $J_{11 \backslash 12}^{(l)}$ will be orthogonal to $\mathrm{H}_{12} X_{2}$ for any $X_{2}$ since each $J_{11 \backslash 12}^{(l)} \in \mathcal{R}_{11 \backslash 12}$, and $\mathrm{H}_{12} X_{2} \in \mathcal{R}_{12}$ for any $X_{2}$, and $\mathcal{R}_{11 \backslash 12}$ is the orthogonal complement of $\mathcal{R}_{12}$. Therefore, each $\xi_{1}^{(l)}$ will be interference free, i.e., will be a linear combination of the symbols $\left\{\chi_{1}^{(l)}\right\}_{l=1}^{d_{1}^{\prime}}$ plus noise, and will contain no contribution from the $\left\{\chi_{2}^{(l)}\right\}_{l=1}^{d_{2}^{\prime}}$ symbols. One can check that these $\operatorname{dim} \mathcal{R}_{11 \backslash 12}$ projections result in

$$
\begin{aligned}
\xi_{1}^{(l)} & =\sum_{m=1}^{d_{1}^{\prime}} \sigma_{11}^{(l)}\left\langle U_{11}^{(m)}, J_{11 \backslash 12}^{(l)}\right\rangle \chi_{1}^{(m)}+\left\langle Z_{1}, J_{11 \backslash 12}^{(l)}\right\rangle, \\
l & =1,2, \ldots, \operatorname{dim} \mathcal{R}_{11 \backslash 12 .}
\end{aligned}
$$

It remains to obtain $d_{1}^{\prime}-\operatorname{dim} \mathcal{R}_{11 \backslash 12}=\operatorname{dim} \mathcal{R}_{11}-$ $\operatorname{dim} \mathcal{R}_{11 \backslash 12}=\operatorname{dim} \mathcal{R}_{11 \cap 12}$ more independent and interference-free linear combinations of $T_{1}$ 's symbols so that $R_{1}$ can solve the system and recover the symbols. Receiver $R_{1}$ will obtain these linear combinations via a careful projection onto a subspace of $\mathcal{R}_{12}$ (which is the orthogonal complement of $\mathcal{R}_{11 \backslash 12}$, the space onto which we have already projected $Y_{1}$ to obtain the first $\operatorname{dim} \mathcal{R}_{11 \backslash 12}$ linear combinations). Recall that the set of left-singular functions of $\mathrm{H}_{12},\left\{U_{12}^{(l)}\right\}_{l=1}^{\operatorname{dim} \mathcal{R}_{12}}$, form an orthonormal basis for $\mathcal{R}_{12}$. Receiver $R_{1}$ obtains the remaining $\mathcal{R}_{11 \cap 12}$ linear combinations by projecting $Y_{1}$ onto the last $\operatorname{dim} \mathcal{R}_{11 \cap 12}$ of these basis functions, forming $\left\{\xi_{1}^{(l)}\right\}_{l=\operatorname{dim} \mathcal{R}_{11 \backslash 12}+1}^{\operatorname{dim} \mathcal{R}_{11}}$ by computing

$$
\begin{aligned}
\xi_{1}^{\left(k+\operatorname{dim} \mathcal{R}_{11 \backslash 12}\right)}= & \left\langle Y_{1}, U_{12}^{\left(\operatorname{dim} \mathcal{R}_{12}-k\right)}\right\rangle, \\
& k=0,1, \ldots, \operatorname{dim} \mathcal{R}_{11 \cap 12}-1, \\
= & \left\langle\mathrm{H}_{11} X_{1}+\mathrm{H}_{12} X_{2}+Z_{1}, U_{12}^{\left(\operatorname{dim} \mathcal{R}_{12}-k\right)}\right\rangle \\
= & \left\langle\mathrm{H}_{11} X_{1}, U_{12}^{\left(\operatorname{dim} \mathcal{R}_{12}-k\right)}\right\rangle \\
& +\left\langle\mathrm{H}_{12} X_{2}, U_{12}^{\left(\operatorname{dim} \mathcal{R}_{12}-k\right)}\right\rangle \\
& +\left\langle Z_{1}, U_{12}^{\left(\operatorname{dim} \mathcal{R}_{12}-k\right)}\right\rangle .
\end{aligned}
$$

We compute the terms of Eq. (64) individually. The contribution of $T_{1}$ 's transmit wavevector is

$$
\begin{aligned}
\left\langle\mathrm{H}_{11} X_{1}, U_{12}^{\left(\operatorname{dim} \mathcal{R}_{12}-k\right)}\right\rangle & \left\langle\sum_{m=1}^{d_{1}^{\prime}} \sigma_{11}^{(m)} U_{11}^{(m)}\left\langle V_{11}^{(m)}, X_{1}\right\rangle, U_{12}^{\left(\operatorname{dim} \mathcal{R}_{12}-k\right)}\right\rangle \\
= & \left\langle\sum_{m=1}^{d_{1}^{\prime}} \sigma_{11}^{(m)} U_{11}^{(m)}\left\langle V_{11}^{(m)}, \sum_{i=1}^{d_{1}^{\prime}} \chi_{1}^{(i)} V_{11}^{(i)}\right\rangle, U_{12}^{\left(\operatorname{dim} \mathcal{R}_{12}-k\right)}\right\rangle \\
= & \sum_{m=1}^{d_{1}^{\prime}} \sigma_{11}^{(m)}\left\langle U_{11}^{(m)}, U_{12}^{\left(\operatorname{dim} \mathcal{R}_{12}-k\right)}\right\rangle \chi_{1}^{(m)}, \quad k=0,1, \ldots, \\
& \operatorname{dim} \mathcal{R}_{11 \cap 12}-1 .
\end{aligned}
$$

In the first step, (65), we use the singular function decomposition of $\mathrm{H}_{11}$. In the second step, (66), we plug in the construction of $X_{1}$ given in 46, and in the last step, (67), we leverage the fact that $\sum_{i} \chi_{1}^{(i)}\left\langle V_{11}^{(m)}, V_{11}^{(i)}\right\rangle=\chi_{1}^{(m)}$, due to the orthonormality of the right singular functions. The contribution of $T_{2}$ 's interfering wavevector is

$$
\begin{aligned}
& \left\langle\mathrm{H}_{12} X_{2}, U_{12}^{\left(\mathrm{dim} \mathcal{R}_{12}-k\right)}\right\rangle \\
& =\left\langle\mathrm{H}_{12}\left(X_{2_{\mathrm{Orth}}}+X_{2_{\text {Int }}}\right), U_{12}^{\left(\mathrm{dim} \mathcal{R}_{12}-k\right)}\right\rangle \\
& =\left\langle\mathrm{H}_{12} X_{2_{\mathrm{lnt}}}, U_{12}^{\left(\mathrm{dim} \mathcal{R}_{12}-k\right)}\right\rangle \\
& =\left\langle\sum_{m=1}^{\operatorname{dim} \mathcal{T}_{12}} \sigma_{12}^{(m)} U_{12}^{(m)}\left\langle V_{12}^{(m)}, X_{2 \text { Int }}\right\rangle,\right. \\
& \left.U_{12}^{\left(\operatorname{dim} \mathcal{R}_{12}-k\right)}\right) \\
& =\left\langle\sum_{m=1}^{\operatorname{dim} \mathcal{T}_{12}} \sigma_{12}^{(m)} U_{12}^{(m)}\left\langle V_{12}^{(m)}, \sum_{i=1}^{d_{2 \operatorname{lnt}}^{\prime}} \chi_{2}^{\left(i+d_{2 \mathrm{Orhh}}^{\prime}\right)} V_{12}^{(i)}\right\rangle,\right. \\
& \left.U_{12}^{\left(\operatorname{dim} \mathcal{R}_{12}-k\right)}\right\rangle \\
& =\left\langle\sum_{i=1}^{d_{\text {Int }}^{\prime}} \chi_{2}^{\left(i+d_{2 \text { Orth }}^{\prime}\right.}\right)^{\operatorname{dim} \mathcal{T}_{12}} \sum_{m=1}^{(m)} U_{12}^{(m)}\left\langle V_{12}^{(m)}, V_{12}^{(i)}\right\rangle, \\
& \left.U_{12}^{\left(\operatorname{dim} \mathcal{R}_{12}-k\right)}\right\rangle \\
& =\sum_{i=1}^{d_{2 \mathrm{lnt}}^{\prime}} \chi_{2}^{\left(i+d_{2 \mathrm{Orh}}^{\prime}\right)} \sigma_{12}^{(i)}\left\langle U_{12}^{(i)}, U_{12}^{\left(\mathrm{dim} \mathcal{R}_{12}-k\right)}\right\rangle \text {, } \\
& =\sum_{i=1}^{d_{2 \operatorname{lnt}}^{\prime}} \chi_{2}^{\left(i+d_{2}^{\prime}{ }_{\text {Orth }}\right)} \sigma_{12}^{(i)} \delta_{\left(i, \operatorname{dim} \mathcal{R}_{12}-k\right)} \text {, } \\
& =0, \quad k=0,1, \ldots, \operatorname{dim} \mathcal{R}_{11 \cap 12}-1,
\end{aligned}
$$


In step (68) above, we use the fact that $\mathrm{H}_{12} X_{2 \text { orth }}=0$ by the construction of $X_{2 \text { orth }}$. Step (69) uses the singular function expansion of $\mathrm{H}_{12}$, step (70) substitutes the construction of $X_{2 \operatorname{lnt}}$, step (71) rearranges terms and step (72) leverages the orthonormality of the right singular functions. In the last step, (74), we have leveraged that when $\operatorname{dim} \mathcal{T}_{12} \leq \operatorname{dim} \mathcal{R}_{12}, d_{2 \text { Int }}^{\prime} \leq \operatorname{dim} \mathcal{R}_{12 \backslash 11}$ (see Eq. 50), which means the largest value of $i$ in the summation, $d_{2 \mid n t}^{\prime}$, is smaller that the smallest value of $\operatorname{dim} \mathcal{R}_{12}-k$ under consideration, $\operatorname{dim} \mathcal{R}_{12}-\mathcal{R}_{11 \cap 12}+1=\operatorname{dim} \mathcal{R}_{12 \backslash 11}+1$, so that the delta-function $\delta_{\left(i, \operatorname{dim} \mathcal{R}_{12}-k\right)}$ will never evaluate to one. Substituting (67) and (74) back into (64) shows that the output symbols obtained by projecting $Y_{1}$ onto the last $\mathcal{R}_{11 \cap 12}$ functions of $\left\{U_{12}^{(l)}\right\}_{l=1}^{\operatorname{dim} \mathcal{R}_{12}}$ are

$$
\begin{aligned}
\xi_{1}^{\left(k+\operatorname{dim} \mathcal{R}_{11 \backslash 12}\right)}= & \sum_{m=1}^{d_{1}^{\prime}} \sigma_{11}^{(m)}\left\langle U_{11}^{(m)}, U_{12}^{\left(\operatorname{dim} \mathcal{R}_{12}-k\right)}\right\rangle \chi_{1}^{(m)} \\
& +\left\langle Z_{1}, U_{12}^{\left(\operatorname{dim} \mathcal{R}_{12}-k\right)}\right\rangle, k=0,1, \ldots, \operatorname{dim} \mathcal{R}_{11 \cap 12}-1 .
\end{aligned}
$$

Combining the processing in all cases, we see that receiver $R_{1}$ has formed a set of $d_{1}^{\prime}$ complex scalars $\left\{\xi_{1}^{(l)}\right\}_{l=1}^{d_{1}^{\prime}}$, such that

$$
\xi_{1}^{(l)}=\sum_{m=1}^{d_{1}^{\prime}} a_{1}^{(l m)} \chi_{1}^{(m)}+\zeta_{1}^{(l)}, \quad l=1,2, \ldots, d_{1}^{\prime},
$$

where

$$
\begin{aligned}
& a_{1}^{(l m)} \\
& =\left\{\begin{array}{lr}
\delta_{l m} \sigma_{11}^{(l)} & : \operatorname{dim} \mathcal{T}_{12}>\operatorname{dim} \mathcal{R}_{12} \\
\sigma_{11}^{(m)}\left\langle U_{11}^{(m)}, J_{11 \backslash 12}^{(l)}\right\rangle & : \operatorname{dim} \mathcal{T}_{12} \leq \operatorname{dim} \mathcal{R}_{12}, l \leq \operatorname{dim} \mathcal{R}_{11 \backslash 12}, \\
\sigma_{11}^{(m)}\left\langle U_{11}^{(m)}, U_{12}^{\left(\operatorname{dim} \mathcal{R}_{12}+\operatorname{dim} \mathcal{R}_{11 \backslash 12}-l\right)}\right\rangle & : \operatorname{dim} \mathcal{T}_{12} \leq \operatorname{dim} \mathcal{R}_{12}, l>\operatorname{dim} \mathcal{R}_{11 \backslash 12}
\end{array}\right.
\end{aligned}
$$

and

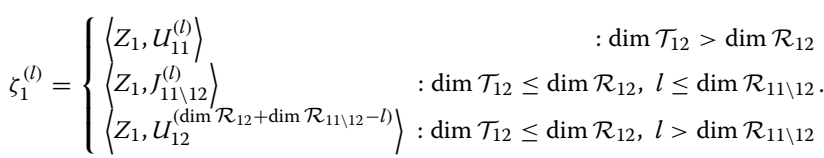

Thus, as desired, in all cases the base station receiver $R_{1}$ is able to obtain $d_{1}^{\prime}$ interference-free linear combinations of the $d_{1}^{\prime}$ symbols from the uplink user transmitter $T_{1}$. Now, we move to the processing at the downlink user receiver.

Processing at $R_{2}$ : We wish to show that the downlink receiver, $R_{2}$, can recover the $d_{2}^{\prime}$ symbols transmitted by the base station transmitter, $T_{w}$. Let $\left\{\sigma_{22}^{(k)}, U_{22}^{(k)}, V_{22}^{(k)}\right\}$ be a singular system for the operator $\mathrm{H}_{22}$, and let $r_{22} \equiv \min \left\{2 L_{T_{2}}\left|\Psi_{T_{22}}\right|, 2 L_{R_{2}}\left|\Psi_{R_{22}}\right|\right\}$. From Property 2 of
Lemma 1 , we know that $\sigma_{22}^{(k)}$ is zero for all $k>r_{22}$ and nonzero for $k \leq r_{22}$, so that

$$
Y_{2}=\mathrm{H}_{22} X_{2}+Z_{2}=\sum_{k=1}^{r_{22}} \sigma_{22}^{(k)} U_{22}^{(k)}\left\langle V_{22}^{(k)}, X_{2}\right\rangle+Z_{2}
$$

One can check that

$\xi_{2}^{(l)}=\left\langle U_{22}^{(l)}, Y_{2}\right\rangle=\sum_{m=1}^{d_{2}^{\prime}} a_{2}^{(l m)} \chi_{2}^{(m)}+\zeta_{2}^{(l)}, \quad l=1, \ldots, d_{2}^{\prime}$,

where

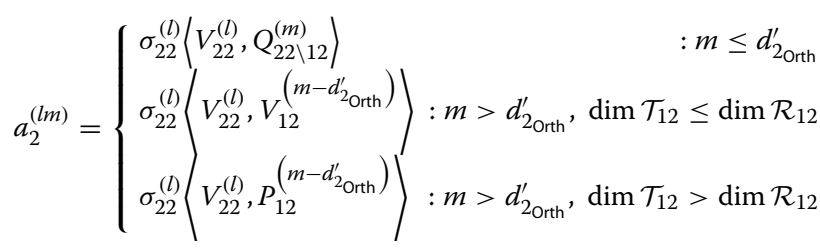

and

$$
\zeta_{2}^{(l)}=\left\langle U_{22}^{(l)}, Z_{2}\right\rangle
$$

\subsubsection{Reducing to parallel point-to-point vector channels}

The above processing at each transmitter and receiver has allowed the receivers $R_{1}$ and $R_{2}$ to recover the symbols

$$
\begin{array}{ll}
\xi_{1}^{(l)}=\sum_{m=1}^{d_{1}^{\prime}} a_{1}^{(l m)} \chi_{1}^{(m)}+\zeta_{1}^{(l)}, & l=1,2, \ldots, d_{1}^{\prime}, \\
\xi_{2}^{(l)}=\sum_{m=1}^{d_{2}^{\prime}} a_{2}^{(l m)} \chi_{2}^{(m)}+\zeta_{2}^{(l)}, & l=1, \ldots, d_{2}^{\prime},
\end{array}
$$

respectively, where the linear combination coefficients, $a_{1}^{(l m)}$ and $a_{2}^{(l m)}$, are given in (77) and (81), respectively, and the additive noise on each of the recovered symbols, $\zeta_{1}^{(l)}$ and $\zeta_{2}^{(l)}$, are given in (78) and (82), respectively.

We can rewrite (83-84) in matrix notation as

$$
\begin{aligned}
& \xi_{1}=A_{1} \chi_{1}+\zeta_{1}, \\
& \xi_{2}=A_{2} \chi_{2}+\zeta_{2},
\end{aligned}
$$

where $\chi_{1}$ and $\chi_{2}$ are the $d_{1}^{\prime} \times 1$ and $d_{2}^{\prime} \times 1$ vectors of input symbols for transmitters $T_{1}$ and $T_{2}$, respectively, $\zeta_{1}$ and $\zeta_{2}$ are the $d_{1}^{\prime} \times 1$ and $d_{2}^{\prime} \times 1$ vectors of additive noise, respectively, and $\boldsymbol{A}_{1}$ and $\boldsymbol{A}_{2}$ are $d_{1}^{\prime} \times d_{1}^{\prime}$ and $d_{2}^{\prime} \times d_{2}^{\prime}$ square matrices whose elements are taken from $a_{1}^{(l m)}$ and $a_{2}^{(l m)}$, respectively. The matrices $A_{1}$ and $A_{2}$ will be full rank for all but a measure-zero set of channel response kernels. Also, since each of the $\zeta_{j}^{(l)}$ 's are linear combinations of Gaussian random variables, the the noise vectors, $\zeta_{1}$ and $\zeta_{2}$, are Gaussian distributed. Therefore, the spatial 
processing has reduced the original channel to two parallel full-rank Gaussian vector channels: the first a $d_{1}^{\prime} \times d_{1}^{\prime}$ channel and the second a $d_{2}^{\prime} \times d_{2}^{\prime}$ channel, which are well known to have $d_{1}^{\prime}$ and $d_{2}^{\prime}$ degrees-of-freedom, respectively [34]. Therefore, the spatial degrees-of-freedom pair $\left(d_{1}^{\prime}, d_{2}^{\prime}\right)$ is indeed achievable.

Lemma 3 The degree-of-freedom pairs $\left(d_{1}^{\prime}, d_{2}^{\prime}\right)$ and $\left(d_{1}^{\prime \prime}, d_{2}^{\prime \prime}\right)$, are the corner points of $\mathcal{D}_{\mathrm{FD}}$, that is

$$
\begin{aligned}
& \left(d_{1}^{\prime}, d_{2}^{\prime}\right)=\left(d_{1}^{\max }, \min \left\{d_{2}^{\max }, d_{\mathrm{sum}}^{\max }-d_{1}^{\max }\right\}\right) \\
& \left(d_{1}^{\prime \prime}, d_{2}^{\prime \prime}\right)=\left(\min \left\{d_{1}^{\max }, d_{\mathrm{sum}}^{\max }-d_{2}^{\max }\right\}, d_{2}^{\max }\right) .
\end{aligned}
$$

Proof Note that it is sufficient to prove only Eq. (87), as Eq. (88) follows by the symmetry of the expressions. It is easy to see that $d_{1}^{\prime}=\min$ $\left\{2 L_{T_{1}}\left|\Psi_{T_{11}}\right|, 2 L_{R_{1}}\left|\Psi_{R_{11}}\right|\right\}=d_{1}^{\max }$, but it is not so obvious that $d_{2}^{\prime}=\min \left\{d_{2}^{\max }, d_{\text {sum }}^{\max }-d_{1}^{\max }\right\}$. However, one can verify that $d_{2}^{\prime}=\min \left\{d_{2}^{\max }, d_{\text {sum }}^{\max }-d_{1}^{\max }\right\}$ by evaluating the left- and right-hand sides for all combinations of the conditions

$$
\begin{aligned}
& L_{T_{1}}\left|\Psi_{T_{11}}\right| \lesseqgtr L_{R_{1}}\left|\Psi_{R_{11}}\right|, \\
& L_{T_{2}}\left|\Psi_{T_{12}}\right| \lesseqgtr L_{R_{1}}\left|\Psi_{R_{12}}\right|
\end{aligned}
$$

and observing equality in each of the four cases. Table 1 shows the expressions to which $d_{2}^{\prime}$ and $\min \left\{d_{2}^{\max }, d_{\text {sum }}^{\max }-d_{1}^{\max }\right\}$ both simplify in each of the four possible cases.

Lemmas 2 and 3 show that the corner points of $\mathcal{D}_{\mathrm{FD}}$, $\left(d_{1}^{\prime}, d_{2}^{\prime}\right)$ and $\left(d_{1}^{\prime \prime}, d_{2}^{\prime \prime}\right)$ are achievable. And, thus, all other points within $\mathcal{D}_{\mathrm{FD}}$ are achievable via time sharing between the schemes that achieve the corner points.

\subsection{Converse}

To establish the converse part of Theorem 1, we must show that the region $\mathcal{D}_{\mathrm{FD}}$, which we have already shown

Table 1 Verifying that the corner points of inner and outer bounds coincide

\begin{tabular}{lc}
\hline Case & $d_{2}^{\prime}=\min \left\{d_{2}^{\max }, d_{\text {sum }}^{\max }-d_{1}^{\max }\right\}$ \\
\hline$L_{T_{1}}\left|\Psi_{T_{11}}\right| \geq L_{R_{1}}\left|\Psi_{R_{11}}\right|$, & $\min \left\{d_{2}^{\max }, 2 L_{T_{2}}\left|\Psi_{T_{22}} \cup \Psi_{T_{12}}\right|\right.$ \\
$L_{T_{2}}\left|\Psi_{T_{12}}\right| \geq L_{R_{1}}\left|\Psi_{R_{12}}\right|$ & $\left.-2 L_{R_{1}}\left|\Psi_{R_{11}} \cap \Psi_{R_{12}}\right|\right\}$ \\
$L_{T_{1}}\left|\Psi_{T_{11}}\right| \geq L_{R_{1}}\left|\Psi_{R_{11}}\right|$, & $\min \left\{d_{2}^{\max }, 2 L_{T_{2}}\left|\Psi_{T_{22} 2} \backslash \Psi_{T_{12}}\right|\right.$ \\
$L_{T_{2}}\left|\Psi_{T_{12}}\right|<L_{R_{1}}\left|\Psi_{R_{12}}\right|$ & $\left.+2 L_{R_{1}}\left|\Psi_{R_{12}}\right| \Psi_{R_{11}} \mid\right\}$ \\
$L_{T_{1}}\left|\Psi_{T_{11}}\right|<L_{R_{1}}\left|\Psi_{R_{11}}\right|$, & $\min \left\{d_{2}^{\max }, 2 L_{T_{2}}\left|\Psi_{T_{22}} \cup \Psi_{T_{12}}\right|\right.$ \\
$L_{T_{2}}\left|\Psi_{T_{12}}\right| \geq L_{R_{1}}\left|\Psi_{R_{12}}\right|$ & $\left.+2 L_{R_{1}}\left|\Psi_{R_{11}} \backslash \Psi_{R_{12}}\right|-2 L_{T_{1}}\left|\Psi_{T_{11}}\right|\right\}$ \\
$L_{T_{1}}\left|\Psi_{T_{11}}\right|<L_{R_{1}}\left|\Psi_{R_{11}}\right|$, & \\
$L_{T_{2}}\left|\Psi_{T_{12}}\right|<L_{R_{1}}\left|\Psi_{R_{12}}\right|$ & $\min \left\{d_{2}^{\max }, 2 L_{T_{2}}\left|\Psi_{T_{22}} \backslash \Psi_{T_{12}}\right|\right.$ \\
\hline
\end{tabular}

is achievable, is also an outer bound on the degrees-offreedom, i.e., we want to show that if an arbitrary degreeof-freedom pair $\left(d_{1}, d_{2}\right)$ is achievable, then $\left(d_{1}, d_{2}\right) \in \mathcal{D}_{\mathrm{FD}}$. It is easy to see that if $\left(d_{1}, d_{2}\right)$ is achievable, then the singe-user constraints on $\mathcal{D}_{\mathrm{FD}}$, given in (14) and (15), must be satisfied as the degrees-of-freedom for each flow cannot be more than the point-to-point degrees-offreedom shown in [24]. Thus, the only step remaining in the converse is to establish an outer bound on the sum degrees-of-freedom which coincides with $d_{\text {sum }}^{\max }$, the sumdegrees-of-freedom constraint on the achievable region, $\mathcal{D}_{\mathrm{FD}}$, given in (16).

Thus, to conclude the converse argument, we will now prove the following Genie-aided outer bound on the sum degrees-of-freedom which coincides with the sumdegrees-of-freedom constraint on the achievable region.

\section{Lemma 4}

$$
\begin{aligned}
d_{1}+d_{2} \leq d_{\text {sum }}^{\max }= & 2 L_{T_{2}}\left|\Psi_{T_{22}} \backslash \Psi_{T_{12}}\right|+2 L_{R_{1}}\left|\Psi_{R_{11}} \backslash \Psi_{R_{12}}\right| \\
& +2 \max \left(L_{T_{2}}\left|\Psi_{T_{12}}\right|, L_{R_{1}}\left|\Psi_{R_{12}}\right|\right) .
\end{aligned}
$$

Sketch of proof: Before diving into the full proof, we would first like to give a brief overview of the steps in the converse proof. Our process for proving Lemma 4 is twofold.

1) First, a genie expands the transmit scattering intervals $\Psi_{T_{22}}$ and $\Psi_{T_{12}}$ until the two intervals are fully overlapped, and likewise expands expands $\Psi_{R_{11}}$ and $\Psi_{R_{12}}$ until they are fully overlapped, as shown in Fig. 6. To ensure that the net manipulation of the genie can only enlarge $\mathcal{D}_{\mathrm{FD}}$, the genie also increases the array lengths $L_{T_{2}}$ and $L_{R_{1}}$ sufficiently for any added interference due to the expansion of $\Psi_{T_{12}}$ and

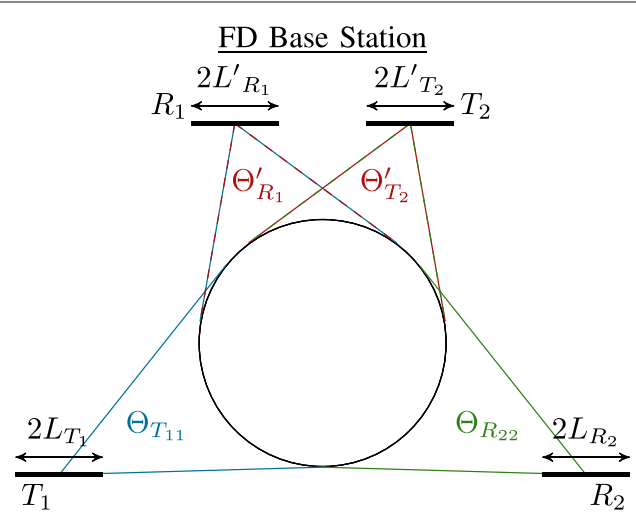

User 1 (Uplink)

User 2 (Downlink)

Fig. 6 Genie-aided channel mode 
$\Psi_{R_{12}}$ to be compensated by the increased array lengths.

2) After the above genie manipulation is performed, the maximum of the $T_{2}$ and $R_{1}$ signaling dimensions are equal to $d_{\text {sum }}^{\max }$ in constraint (16), and since the scattering intervals are overlapped, the channel model becomes the Hilbert space equivalent of the well-studied MIMO Z-channel $[35,36]$. The Hilbert space analog to the bounding techniques employed in $[35,36]$ are then leveraged to conclude the converse proof.

Proof We prove Lemma 4 by way of a Genie that aids the transmitters and receivers by enlarging the scattering intervals and lengthening the antenna arrays in a way that can only enlarge the degrees-of-freedom region. Applying the point-to-point bounds to the Genie-aided system in a careful way then establishes the outer bound. Assume an arbitrary scheme achieves the degrees-of-freedom pair $\left(d_{1}, d_{2}\right)$. Thus receivers $R_{1}$ and $R_{2}$ can decode their corresponding messages with probability of error approaching zero. We must show that the assumption of $\left(d_{1}, d_{2}\right)$ being achievable implies the constraint in Eq. (91).

Let a Genie expand both scattering intervals at $T_{2}$ into the union of the two scattering intervals, that is expand $\Psi_{T_{22}}$ and $\Psi_{T_{12}}$ to

$$
\Psi_{T_{22}}^{\prime}=\Psi_{T_{12}}^{\prime}=\Psi_{T_{2}}^{\prime} \equiv \Psi_{T_{22}} \cup \Psi_{T_{12}} .
$$

Likewise, the Genie expands the scattering intervals at $R_{1}$ into their union, that is expand $\Psi_{R_{11}}$ and $\Psi_{R_{12}}$ to

$$
\Psi_{R_{11}}^{\prime}=\Psi_{R_{12}}^{\prime} \equiv \Psi_{R_{1}}^{\prime}=\Psi_{R_{11}} \cup \Psi_{R_{12}} \text {. }
$$

The Genie's expansion of $\Psi_{T_{22}}$ to $\Psi_{T_{2}}^{\prime}$ can only enlarge the degrees-of-freedom region, as $T_{2}$ could simply not transmit in the added interval $\Psi_{T_{2}}^{\prime} \backslash \Psi_{T_{22}}$ (i.e., ignore the added dimensions for signaling to $R_{2}$ ) to obtain the original scenario. Likewise, expanding $\Psi_{R_{11}}$ to $\Psi_{R_{1}}^{\prime}$ will only enlarge the degrees-of-freedom region as $R_{1}$ can ignore the portion of the wavevector received over $\Psi_{R_{1}}^{\prime} \backslash \Psi_{R_{11}}$ to obtain the original scenario. However, expanding the interference scattering clusters, $\Psi_{T_{12}}$ and $\Psi_{R_{12}}$, to $\Psi_{T_{2}}^{\prime}$ and $\Psi_{R_{1}}^{\prime}$, respectively, can indeed shrink the degrees-offreedom region due to the additional interference caused by the added overlap with the signal-of-interest intervals $\Psi_{T_{22}}$ and $\Psi_{R_{22}}$, respectively. We need a final Genie manipulation to compensate for this added interference, so that the net Genie manipulation can only enlarge the degreesof-freedom region. Therefore, in the next step we will have the Genie lengthen the arrays at $T_{2}$ and $R_{1}$ sufficiently to allow any interference introduced by expanding $\Psi_{T_{12}}$ and $\Psi_{R_{12}}$, to $\Psi_{T_{2}}^{\prime}$ and $\Psi_{R_{1}}^{\prime}$, respectively, to be zeroforced without sacrificing any previously available degrees of freedom. Expansion of $\Psi_{T_{12}}$ to $\Psi_{T_{2}}^{\prime} \equiv \Psi_{T_{22}} \cup \Psi_{T_{12}}$ causes the dimension of the interference that $T_{2}$ presents to $R_{1}$ to increase by at most $2 L_{T_{2}}\left|\Psi_{T_{22}} \backslash \Psi_{T_{12}}\right|$. Therefore, let the Genie also lengthen $R_{1}$ 's array from $2 L_{R_{1}}$ to $2 L_{R_{1}}^{\prime}=2 L_{R_{1}}+2 L_{T_{2}} \frac{\left|\Psi_{T_{22}} \backslash \Psi_{T_{11}} \cup \Psi_{R_{12}}\right|}{\mid \Psi_{2}}$, so that the dimension of the total receive space at $R_{1}, \operatorname{dim} \mathcal{R}_{1}$, is increased from $\operatorname{dim} \mathcal{R}_{1}=2 L_{R_{1}}\left|\Psi_{R_{11}} \cup \Psi_{R_{12}}\right|$ to

$$
\begin{aligned}
\operatorname{dim} \mathcal{R}_{1}^{\prime} & =2 L_{R_{1}}^{\prime}\left|\Psi_{R_{11}} \cup \Psi_{R_{12}}\right| \\
& =\left(2 L_{R_{1}}+2 L_{T_{2}} \frac{\left|\Psi_{T_{22}} \backslash \Psi_{T_{12}}\right|}{\left|\Psi_{R_{11}} \cup \Psi_{R_{12}}\right|}\right)\left|\Psi_{R_{11}} \cup \Psi_{R_{12}}\right| \\
& =2 L_{R_{1}}\left|\Psi_{R_{11}} \cup \Psi_{R_{12}}\right|+2 L_{T_{2}}\left|\Psi_{T_{22}} \backslash \Psi_{T_{12}}\right| \\
& =\operatorname{dim} \mathcal{R}_{1}+2 L_{T_{2}}\left|\Psi_{T_{22}} \backslash \Psi_{T_{12}}\right| .
\end{aligned}
$$

We observe in (95), that the Genie's lengthening of the $T_{2}$ array by $2 L_{T_{2}} \frac{\left|\Psi_{T_{22}} \backslash \Psi_{R_{12}} \cup \Psi_{R_{12}}\right|}{\left|{ }_{2}\right|}$ has increased the dimension of $R_{1}$ 's total receive signal space by $2 L_{T_{2}}\left|\Psi_{T_{22}} \backslash \Psi_{T_{12}}\right|$, which is the worst case increase in the dimension of the interference from $T_{2}$ due to expansion of $\Psi_{T_{12}}$ to $\Psi_{T_{22}} \cup \Psi_{T_{12}}$. Therefore, the dimension of the subspace of $\mathcal{R}_{1}^{\prime}$ which is orthogonal to the interference from $T_{2}$ will be at least as large as in the original orthogonal space of $\mathcal{R}_{1}$. Thus, the combined expansion of $\Psi_{T_{12}}$ to $\Psi_{T_{2}}^{\prime}$ and lengthening of the $R_{1}$ array to $L_{R_{1}}^{\prime}$ can only enlarge the degrees-of-freedom region. Analogously, expansion of $\Psi_{R_{12}}$ to $\Psi_{R_{1}}^{\prime} \equiv \Psi_{R_{11}} \cup \Psi_{R_{12}}$ increases the dimension of $\mathcal{R}_{12}$, the subspace of $R_{1}$ 's receive space which is vulnerable to interference from $T_{2}$, by at most $2 L_{R_{1}}\left|\Psi_{R_{11}} \backslash \Psi_{R_{12}}\right|$. Therefore, let the Genie lengthen $T_{2}$ 's array from $2 L_{T_{2}}$ to $2 L^{\prime} T_{2}=2 L_{T_{2}}+2 L_{R_{1}} \frac{\left|\Psi_{R_{11}} \backslash \Psi_{T_{12}} \cup \Psi_{T_{12}}\right|}{\mid \Psi_{2}}$, so that the dimension of the transmit space at $T_{2}, \operatorname{dim} \mathcal{T}_{2}$, is increased from $\operatorname{dim} \mathcal{T}_{2}=2 L_{T_{2}}\left|\Psi_{T_{22}} \cup \Psi_{T_{12}}\right|$ to

$$
\begin{aligned}
\operatorname{dim} \mathcal{T}_{2}^{\prime} & =2 L_{T_{2}}^{\prime}\left|\Psi_{T_{22}} \cup \Psi_{T_{12}}\right| \\
& =\left(2 L_{T_{2}}+2 L_{R_{1}} \frac{\left|\Psi_{R_{11}} \backslash \Psi_{R_{12}}\right|}{\left|\Psi_{T_{22}} \cup \Psi_{T_{12}}\right|}\right)\left|\Psi_{T_{22}} \cup \Psi_{T_{12}}\right| \\
& =2 L_{T_{2}}\left|\Psi_{T_{22}} \cup \Psi_{T_{12}}\right|+2 L_{R_{1}}\left|\Psi_{R_{11}} \backslash \Psi_{R_{12}}\right| \\
& =\operatorname{dim} \mathcal{T}_{2}+2 L_{R_{1}}\left|\Psi_{R_{11}} \backslash \Psi_{R_{12}}\right| .
\end{aligned}
$$

We see in (99) that the Genie's lengthening of $T_{2}$ 's array to $2 L^{\prime} T_{2}$ increases the dimension of $T_{2}$ 's transmit signal space by $2 L_{R_{1}}\left|\Psi_{R_{11}} \backslash \Psi_{R_{12}}\right|$, which is the worst case increase in the dimension of the subspace of $R_{1}$ 's receive subspace vulnerable to interference from $T_{2}$. Therefore, $T_{1}$ can leverage these extra $2 L_{R_{1}}\left|\Psi_{R_{11}} \backslash \Psi_{R_{12}}\right|$ dimensions to zero force to the subspace of $R_{1}$ 's receive space that has become vulnerable to interference from $T_{2}$ due to the expansion $\Psi_{R_{12}}$ to $\Psi_{R_{1}}^{\prime}$. Thus, the net effect of the Genie's expansion of $T_{2}$ 's interference scattering interval, $\Psi_{R_{12}}$, to $\Psi_{R_{1}}^{\prime}$ and lengthening of the $T_{2}$ array to $2 L^{\prime} T_{2}$ can only enlarge the degrees-of-freedom region. 
The Genie-aided channel is illustrated in Fig. 6, which emphasizes the fact that the Genie has made the channel fully-coupled in the sense that the signal-of-interest scattering and the interference scattering intervals are identical: any direction of departure from $T_{2}$ which scatters to $R_{2}$ also scatters to $R_{1}$, and any direction of arrival to $R_{1}$ which signal can be received from $T_{1}$ is a direction from which signal can be received from $T_{2}$. Note that for the Genie-aided channel,

$$
\begin{aligned}
\max & \left(\operatorname{dim} \mathcal{T}_{2}^{\prime}, \operatorname{dim} \mathcal{R}_{1}^{\prime}\right) \\
& =2 \max \left(L_{T_{2}}^{\prime}\left|\Psi_{T_{2}}^{\prime}\right| L_{R_{1}}^{\prime}\left|\Psi_{R_{1}}^{\prime}\right|\right) \\
& =2 \max \left\{\begin{array}{l}
\left(L_{T_{2}}+L_{R_{1}} \frac{\left|\Psi_{R_{11}}\right| \Psi_{R_{12}} \mid}{\left|\Psi_{T_{2}}\right|}\right)\left|\Psi_{T_{2}}^{\prime}\right|, \\
\left(L_{R_{1}}+L_{T_{2}} \frac{\left|\Psi_{T_{22}}\right| \Psi_{T_{12}} \mid}{\left|\Psi_{R_{1}}^{\prime}\right|}\right)\left|\Psi_{R_{1}}^{\prime}\right|
\end{array}\right\} \\
= & 2 \max \left\{\begin{array}{c}
L_{T_{2}}\left|\Psi_{T_{2}}^{\prime}\right|+L_{R_{1}}\left|\Psi_{R_{11}} \backslash \Psi_{R_{12}}\right| \\
L_{R_{1}}\left|\Psi_{R_{1}}^{\prime}\right|+L_{T_{2}}\left|\Psi_{T_{22}} \backslash \Psi_{T_{12}}\right|
\end{array}\right\} \\
= & 2 \max \left\{\begin{array}{c}
L_{T_{2}}\left|\Psi_{T_{22}} \cup \Psi_{T_{12}}\right|+L_{R_{1}}\left|\Psi_{R_{11}} \backslash \Psi_{R_{12}}\right| \\
L_{R_{1}}\left|\Psi_{R_{11}} \cup \Psi_{R_{12}}\right|+L_{T_{2}}\left|\Psi_{T_{22}} \backslash \Psi_{T_{12}}\right|
\end{array}\right\}
\end{aligned}
$$

$$
=2 \max \left\{\begin{array}{c}
L_{T_{2}}\left(\left|\Psi_{T_{12}}\right|+\left|\Psi_{T_{22}} \backslash \Psi_{T_{12}}\right|\right)+L_{R_{1}}\left|\Psi_{R_{11}} \backslash \Psi_{R_{12}}\right| \\
L_{R_{1}}\left(\left|\Psi_{R_{12}}\right|+\left|\Psi_{R_{11}} \backslash \Psi_{R_{12}}\right|\right)+L_{T_{2}}\left|\Psi_{T_{22}} \backslash \Psi_{T_{12}}\right|
\end{array}\right\}
$$

$$
=2 \max \left\{\begin{array}{c}
L_{T_{2}}\left|\Psi_{T_{2}}\right|+L_{T_{2}}\left|\Psi_{T_{22}} \backslash \Psi_{T_{12}}\right|+L_{R_{1}}\left|\Psi_{R_{11}} \backslash \Psi_{R_{12}}\right| \\
L_{R_{1}}\left|\Psi_{R_{12}}\right|+L_{R_{1}}\left|\Psi_{R_{11}} \backslash \Psi_{R_{12}}\right|+L_{T_{2}}\left|\Psi_{T_{22}} \backslash \Psi_{T_{12}}\right|
\end{array}\right\}
$$

$$
\begin{aligned}
= & 2 \max \left(L_{T_{2}}\left|\Psi_{T_{12}}\right|, L_{R_{1}}\left|\Psi_{R_{12}}\right|\right)+2 L_{T_{2}}\left|\Psi_{T_{22}} \backslash \Psi_{T_{12}}\right| \\
& +2 L_{R_{1}}\left|\Psi_{R_{11}} \backslash \Psi_{R_{12}}\right|,
\end{aligned}
$$

which is the outer bound on sum degrees-of-freedom that we wish to prove. Thus, if we can show that for the Genieaided channel

$$
\begin{aligned}
d_{1}+d_{2} & \leq 2 \max \left(L_{T_{2}}^{\prime}\left|\Psi_{T_{2}}^{\prime}\right|, L_{R_{1}}^{\prime}\left|\Psi_{R_{1}}^{\prime}\right|\right) \\
& =\max \left(\operatorname{dim} \mathcal{T}_{2}^{\prime}, \operatorname{dim} \mathcal{R}_{1}^{\prime}\right)
\end{aligned}
$$

then the converse is established. Because the Genie-aided channel is now fully coupled, it is similar to the continuous Hilbert space analog of the full-rank discrete-antennas MIMO $Z$ interference channel. Thus, the remaining steps in the converse argument are inspired by the techniques used in [35-37] for outer bounding the degrees-offreedom of the MIMO interference channel.

Consider the case in which $\operatorname{dim} \mathcal{T}_{2}^{\prime} \leq \operatorname{dim} \mathcal{R}_{1}^{\prime}$. Since our Genie has enforced $\Psi_{T_{22}}^{\prime}=\Psi_{T_{12}}^{\prime}$ and we have assumed $\operatorname{dim} \mathcal{T}_{2}^{\prime} \leq \operatorname{dim} \mathcal{R}_{1}^{\prime}$, receiver $R_{1}$ has access to the entire signal space of $T_{2}$, i.e., $T_{2}$ cannot zero force to $R_{1}$. Moreover, by our hypothesis that $\left(d_{1}, d_{2}\right)$ is achieved, $R_{1}$ can decode the message from $T_{1}$, and can thus reconstruct and subtract the signal received from $T_{1}$ from its received signal.

Since $R_{1}$ has access to the entire signal-space of $T_{2}$, after removing the signal from $T_{1}$ the only barrier to $R_{1}$ also decoding the message from $T_{2}$ is the receiver noise process. If it is not already the case, let a Genie lower the noise at receiver $R_{1}$ until $T_{2}$ has a better channel to $R_{1}$ than $R_{2}$ (this can only increase the capacity region since $R_{1}$ could always locally generate and add noise to obtain the original channel statistics). By hypothesis, $R_{2}$ can decode the message from $T_{2}$, and since $T_{2}$ has a better channel to $R_{1}$ than $R_{2}, R_{1}$ can also decode the message from $T_{1}$.

Since $R_{1}$ can decode the messages from both $T_{1}$ and $T_{2}$, we can bound the degrees-of-freedom region of the Genie-aided channel by the corresponding point-to-point channel in which $T_{1}$ and $T_{2}$ cooperate to jointly communicate their messages to $R_{1}$, which has degrees-of-freedom $\min \left(\operatorname{dim} \mathcal{T}_{1}^{\prime}+\operatorname{dim} \mathcal{T}_{2}^{\prime}, \operatorname{dim} \mathcal{R}_{1}^{\prime}\right)$, which implies that

$$
d_{1}+d_{2} \leq \operatorname{dim} \mathcal{R}_{1}^{\prime}, \quad \text { when } \operatorname{dim} \mathcal{T}_{2}^{\prime} \leq \operatorname{dim} \mathcal{R}_{1}^{\prime} .
$$

Now, consider the alternate case in which $\operatorname{dim} \mathcal{T}_{2}^{\prime}<$ $\operatorname{dim} \mathcal{R}_{1}^{\prime}$. In this case, we let a Genie increase the length of the $R_{1}$ array once more from $2 L_{R_{1}}^{\prime}$ to $2 L^{\prime \prime}{ }_{R_{1}}=$ $2 L^{\prime} T_{2} \frac{\left|\Psi_{T_{2}}^{\prime}\right|}{\left|\Psi_{R_{1}}^{\prime}\right|}>2 L_{R_{1}}^{\prime}$, so that the dimension of the receive signal space at $R_{1}$, which we now call $\mathcal{R}_{1}^{\prime \prime}$, is expanded to

$$
\begin{aligned}
\operatorname{dim} \mathcal{R}_{1}^{\prime \prime} & =2 L_{R_{2}}^{\prime}\left|\Psi_{R_{1}}^{\prime}\right|=\left(2 L_{T_{2}}^{\prime} \frac{\left|\Psi_{T_{2}}^{\prime}\right|}{\left|\Psi_{R_{1}}^{\prime}\right|}\right)\left|\Psi_{R_{1}}^{\prime}\right| \\
& =2 L_{T_{2}}^{\prime}\left|\Psi_{T_{2}}^{\prime}\right|=\operatorname{dim} \mathcal{T}_{2}^{\prime} .
\end{aligned}
$$

Since $\operatorname{dim} \mathcal{R}_{1}^{\prime \prime}=\operatorname{dim} \mathcal{T}_{2}^{\prime}$ and $\Psi_{T_{22}}^{\prime}=\Psi_{T_{12}}^{\prime}, R_{1}$ again has access to the entire transmit signal space of $T_{2}$, we can use the same argument we leveraged above in the $\operatorname{dim} \mathcal{T}_{2}^{\prime} \leq$ $\operatorname{dim} \mathcal{R}_{1}^{\prime}$ case to show that

$d_{1}+d_{2} \leq \operatorname{dim} \mathcal{R}_{1}^{\prime \prime}=\operatorname{dim} \mathcal{T}_{2}^{\prime}, \quad$ when $\operatorname{dim} \mathcal{T}_{2}^{\prime}>\operatorname{dim} \mathcal{R}_{1}^{\prime}$

Combining the bounds in (108) and (111) yields,

$$
\begin{aligned}
d_{1}+d_{2} \leq & \max \left(\operatorname{dim} \mathcal{T}_{2}^{\prime}, \operatorname{dim} \mathcal{R}_{1}^{\prime}\right) \\
= & 2 \max \left(L_{T_{2}}^{\prime}\left|\Psi_{T_{2}}^{\prime}\right|, L_{R_{1}}^{\prime}\left|\Psi_{R_{1}}^{\prime}\right|\right) \\
= & 2 \max \left(L_{T_{2}}\left|\Psi_{T_{12}}\right|, L_{R_{1}}\left|\Psi_{R_{12}}\right|\right) \\
& +2 L_{T_{2}}\left|\Psi_{T_{22}} \backslash \Psi_{T_{12}}\right|+2 L_{R_{1}}\left|\Psi_{R_{11}} \backslash \Psi_{R_{12}}\right|
\end{aligned}
$$

thus showing that the sum-degrees-of-freedom bound of Eq. (16) in Theorem 1 must hold for any achievable degree-of-freedom pair. 
Combining Lemma 4 with the trivial point-to-point bounds establishes that the region $\mathcal{D}_{\mathrm{FD}}$, given in Theorem 1, is an outer bound on any achievable degreesof-freedom pair, thus establishing the converse part of Theorem 1.

\section{Impact on full-duplex design}

We have characterized, $\mathcal{D}_{\mathrm{FD}}$, the degrees-of-freedom region achievable by a full-duplex base-station which uses spatial isolation to avoid self-interference while transmitting the uplink signal while simultaneously receiving. Now, we wish to discuss how this result impacts the operation of full-duplex base stations. In particular, we aim to ascertain in what scenarios full-duplex with spatial isolation outperforms half-duplex, and are there scenarios in which full-duplex with spatial isolation achieves an ideal rectangular degrees-of-freedom region (i.e., both the uplink flow and downlink flow achieving their respective point-to-point degrees-of-freedom).

To answer the above questions, we must first briefly characterize $\mathcal{D}_{\mathrm{HD}}$, the region of degrees-of-freedom pairs achievable via half-duplex mode, i.e., by time-divisionduplex between uplink and downlink transmission. It is easy to see that the half-duplex achievable region is characterized by

$$
\begin{aligned}
& d_{1} \leq \alpha \min \left\{2 L_{T_{1}}\left|\Psi_{T_{11}}\right|, 2 L_{R_{1}}\left|\Psi_{R_{11}}\right|\right\}, \\
& d_{2} \leq(1-\alpha) \min \left\{2 L_{T_{2}}\left|\Psi_{T_{22}}\right|, 2 L_{R_{2}}\left|\Psi_{R_{22}}\right|\right\},
\end{aligned}
$$

where $\alpha \in[0,1]$ is the time sharing parameter. Obviously $\mathcal{D}_{\mathrm{HD}} \subseteq \mathcal{D}_{\mathrm{FD}}$, but we are interested in contrasting the scenarios for which $\mathcal{D}_{\mathrm{HD}} \subset \mathcal{D}_{\mathrm{FD}}$, and full-duplex spatial isolation strictly outperforms half-duplex time division, and the scenarios for which $\mathcal{D}_{\mathrm{HD}}=\mathcal{D}_{\mathrm{FD}}$ and half-duplex can achieve the same performance as full-duplex. We will consider two particularly interesting cases: the fully spread environment, and the symmetric spread environment.

\subsection{Overlapped scattering case}

Consider the worst case for full-duplex operation in which the self-interference backscattering intervals perfectly overlap the forward scattering intervals of the signals-of interest. By "overlapped" we mean that the directions of departure from the base station transmitter, $T_{2}$, that scatter to the intended downlink receiver, $R_{2}$, are identical to the directions of departure that backscatter to the base station receiver, $R_{1}$, as self-interference, so that $\Psi_{T_{11}}=\Psi_{T_{12}}$. Likewise, the directions of arrival to the base station receiver, $R_{1}$, of the intended uplink signal from $T_{1}$ are identical to the directions of arrival of the backscattered self-interference from $T_{2}$, so that $\Psi_{R_{22}}=\Psi_{T_{12}}$. To reduce the number of variables in the degrees-of-freedom expressions, we assume each of the scattering intervals are of size $|\Psi|$, so that
$\left|\Psi_{T_{11}}\right|=\left|\Psi_{R_{11}}\right|=\left|\Psi_{T_{22}}\right|=\left|\Psi_{R_{22}}\right|=\left|\Psi_{T_{12}}\right|=\left|\Psi_{R_{12}}\right| \equiv|\Psi|$. We further assume that the base station arrays are of length $2 L_{R_{1}}=2 L_{T_{2}}=2 L_{\mathrm{BS}}$, and the user arrays are of equal length $2 L_{T_{1}}=2 L_{R_{2}}=2 L_{U s r}$. In this case, the full-duplex degrees-of-freedom region, $\mathcal{D}_{\mathrm{FD}}$, simplifies to

$d_{i} \leq|\Psi| \min \left\{2 L_{\mathrm{BS}}, 2 L_{\mathrm{Usr}}\right\}, i=1,2 ; \quad d_{1}+d_{2} \leq 2 L_{\mathrm{BS}}|\Psi|$

while the half-duplex achievable region, $\mathcal{D}_{\mathrm{HD}}$ simplifies to

$$
d_{1}+d_{2} \leq|\Psi| \min \left\{2 L_{\mathrm{BS}}, 2 L_{\mathrm{Usr}}\right\} .
$$

The following remark characterizes the scenarios for which full-duplex with spatial isolation beats half-duplex.

Remark In the overlapped scattering case, $\mathcal{D}_{\mathrm{HD}} \subset \mathcal{D}_{\mathrm{FD}}$ when $2 L_{\mathrm{BS}}>2 L_{\mathrm{Usr}}$, else $\mathcal{D}_{\mathrm{HD}}=\mathcal{D}_{\mathrm{FD}}$.

We see that full-duplex outperforms half-duplex only if the base station arrays are longer than the user arrays. This is because in the overlapped scattering case, the only way to spatially isolate the self-interference is zero forcing, and zero forcing requires extra antenna resources at the base station. When $2 L_{\mathrm{BS}} \leq 2 L_{\mathrm{Usr}}$, the base station has no extra antenna resources it can leverage for zero forcing, and thus, spatial isolation of the self-inference is no better than isolation via time division. However, when $2 L_{\mathrm{BS}}>2 L_{\mathrm{Usr}}$, the base station transmitter can transmit $\left(2 L_{\mathrm{BS}}-2 L_{\mathrm{Usr}}\right)|\Psi|$ zero-forced streams on the downlink without impeding the reception of the the full $2 L_{U_{s r}}|\Psi|$ streams on the uplink, enabling a sum-degreesof-freedom gain of $\left(2 L_{\mathrm{BS}}-2 L_{\mathrm{Usr}}\right)|\Psi|$ over half-duplex. Indeed when the base station arrays are at least twice as long as the user arrays, the degrees-of-freedom region is rectangular, and both uplink and downlink achieve the ideal $2 L_{U s r}|\Psi|$ degrees-of-freedom.

\subsection{Symmetric spread}

The previous overlapped scattering case is the worst case for full duplex operation. Let us now consider the more general case where the self-interference backscattering and the signal-of-interest forward scattering are not perfectly overlapped. This case illustrates the impact of the overlap of the scattering intervals on full-duplex performance. Once again, to reduce the number of variables, we will make following symmetry assumptions. Assume all the arrays in the network, the two arrays on the base station as well as the array on each of the user devices, are of the same length $2 L$, that is $2 L_{T_{1}}=2 L_{R_{1}}=2 L_{T_{2}}=2 L_{R_{2}}$ 三 $2 L$. Also, assume that the size of the forward scattering intervals to/from the intended receiver/transmitter is the same for all arrays $\left|\Psi_{T_{11}}\right|=\left|\Psi_{R_{11}}\right|=\left|\Psi_{T_{22}}\right|=\left|\Psi_{R_{22}}\right| \equiv$ $\left|\Psi_{\text {Fwd }}\right|$, and that the size of the backscattering interval is the same at the base station receiver as at the base station trasmitter $\left|\Psi_{T_{12}}\right|=\left|\Psi_{R_{12}}\right| \equiv\left|\Psi_{\text {Back }}\right|$. Finally, assume 
the amount of overlap between the backscattering and the forward scattering is the same at the base station transmitter as at the base station receiver so that $\left|\Psi_{T_{22}} \cap \Psi_{T_{12}}\right|=$ $\left|\Psi_{R_{11}} \cap \Psi_{R_{12}}\right| \equiv\left|\Psi_{\text {Fwd }} \cap \Psi_{\text {Back }}\right|=\left|\Psi_{\text {Fwd }}\right|-\left|\Psi_{\text {Fwd }} \backslash \Psi_{\text {Back }}\right|$.

We call $\Psi_{\text {Back }}$ the backscatter interval since it is the angle subtended at the base station by the back-scattering clusters, while we call $\Psi_{\text {Fwd }}$ the forward interval, since it is the angle subtended by the clusters that scatter towards the intended transmitter/receiver. In this case, the full-duplex degree-of-freedom region, $\mathcal{D}_{\mathrm{FD}}$ simplifies to

$$
\begin{aligned}
d_{i} & \leq 2 L\left|\Psi_{\text {Fwd }}\right|, i=1,2 \\
d_{1}+d_{2} & \leq 2 L\left(2\left|\Psi_{\text {Fwd }} \backslash \Psi_{\text {Back }}\right|+\left|\Psi_{\text {Back }}\right|\right)
\end{aligned}
$$

while the half-duplex achievable region, $\mathcal{D}_{\mathrm{HD}}$ is

$$
d_{1}+d_{2} \leq 2 L\left|\Psi_{\mathrm{Fwd}}\right|
$$

Remark Comparing $\mathcal{D}_{\mathrm{FD}}$ and $\mathcal{D}_{\mathrm{HD}}$ above we see that in the case of symmetric scattering, $\mathcal{D}_{\mathrm{HD}}=\mathcal{D}_{\mathrm{FD}}$ if and only if $\Psi_{\mathrm{Fwd}}=\Psi_{\mathrm{Back}}$ else $\mathcal{D}_{\mathrm{HD}} \subset \mathcal{D}_{\mathrm{FD}}$ (we are neglecting the trivial case of $L=0$ ).

Thus, the full-duplex spatial isolation region is strictly larger than the half-duplex time-division region unless the forward interval and the backscattering interval are perfectly overlapped. The intuition is that when $\Psi_{\mathrm{Fwd}}=$ $\Psi_{\text {Back }}$ the scattering interval is shared resource, just as is time, thus trading spatial resources is equivalent to trading time-slots. However, if $\Psi_{\text {Fwd }} \neq \Psi_{\text {Back }}$, there is a portion of space exclusive to each user which can be leveraged to improve upon time division. Moreover, inspection of $\mathcal{D}_{\mathrm{FD}}$ above leads to the following remark.

Remark In the case of symmetric scattering, the degreesof-freedom region is rectangular if and only if

$$
\left|\Psi_{\text {Back }} \backslash \Psi_{\text {Fwd }}\right| \geq\left|\Psi_{\text {Fwd }} \cap \Psi_{\text {Back }}\right| .
$$

The above remark can be verified by comparing (118) and (119) observing that the sum-rate bound, (119), is only active when

$$
2\left|\Psi_{\text {Fwd }} \backslash \Psi_{\text {Back }}\right|+\left|\Psi_{\text {Back }}\right| \geq 2\left|\Psi_{\text {Fwd }}\right|
$$

Straightforward set-algebraic manipulation of condition (122) shows that it is equivalent to (121).

The intuition is that because $\Psi_{\text {Back }} \backslash \Psi_{\text {Fwd }}$ are the set directions in which the base station couples to itself but not to the users, the corresponding $2 L\left|\Psi_{\text {Back }} \backslash \Psi_{\text {Fwd }}\right|$ dimensions are useless for spatial multiplexing, and therefore "free" for zero forcing the self-interference, which has maximum dimension $2 L\left|\Psi_{\text {Fwd }} \cap \Psi_{\text {Back }}\right|$. Thus, when $\left|\Psi_{\text {Back }}\right| \Psi_{\text {Fwd }}|\geq| \Psi_{\text {Fwd }} \cap \Psi_{\text {Back }} \mid$, we can zero force any self-interference that is generated, without sacrificing any resource needed for spatial multiplexing to intended users.

Consider a numerical example in which $\left|\Psi_{\text {Fwd }}\right|=1$ and $\left|\Psi_{\text {Back }}\right|=1$, thus the overlap between the two, $\mid \Psi_{\text {Fwd }} \cap$ $\Psi_{\text {Back }}$, can vary from zero to one. Figure 7 plots the halfduplex region, $\mathcal{D}_{\mathrm{HD}}$, and the full-duplex region, $\mathcal{D}_{\mathrm{FD}}$, for several different values of overlap, $\left|\Psi_{\text {Fwd }} \cap \Psi_{\text {Back }}\right|$. We see that when $\Psi_{\text {Fwd }}=\Psi_{\text {Back }}$ so that $\left|\Psi_{\text {Fwd }} \cap \Psi_{\text {Back }}\right|=1$, both $\mathcal{D}_{\mathrm{HD}}$ and $\mathcal{D}_{\mathrm{FD}}$ are the same triangular region. When $\left|\Psi_{\text {Fwd }} \cap \Psi_{\text {Back }}\right|=0.75$, we get a rectangular region. Once $\left|\Psi_{\text {Fwd }} \cap \Psi_{\text {Back }}\right| \leq 0.5,\left|\Psi_{\text {Back }} \backslash \Psi_{\text {Fwd }}\right|$ becomes greater than 0.5 , such that condition of (121) is satisfied and the degree-of-freedom region becomes rectangular.

The overall takeaway is that as the amount of backscattering increases, more degrees of freedom must be sacrificed to achieve sufficient self-interference suppression, and less degrees of freedom are left for signaling to the desired users. Therefore, full-duplex operation, where self-interference is suppressed by beamforming, is indeed feasible, when the antenna array at the base station is sufficiently large, and when the backscattering is sufficiently limited.

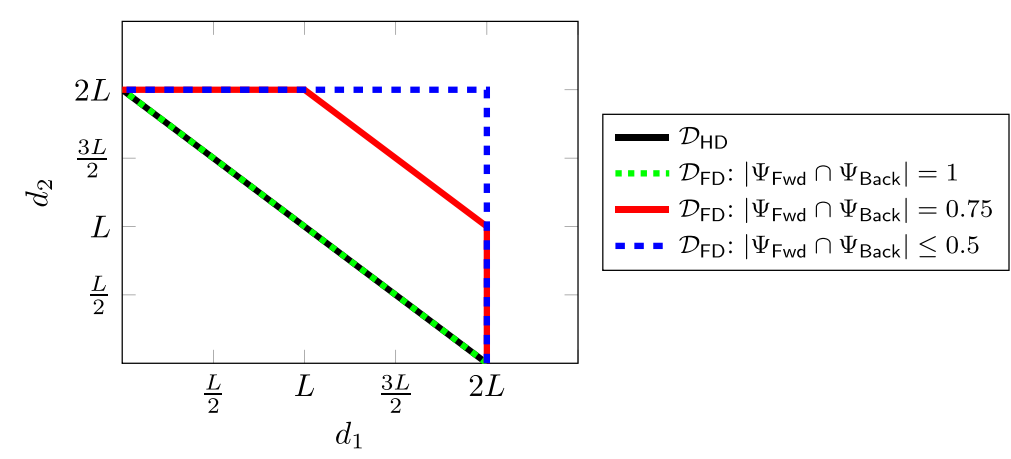

Fig. 7 Symmetric-spread degree-of-freedom regions for different amounts of scattering overlap 


\subsection{Simulation example}

We now consider a simple simulation example that illustrates the results of Theorem 1. In particular, this example illustrates that as the angular spread of the backscattering increases, more transmit degrees of freedom must be sacrificed in order to sufficiently suppress self-interference at the base station. Theorem 1 was derived under several theoretical assumptions which are relaxed in this simulation to show that the same trends still apply. The channel model of (2) focuses on backscattering only; in this simulation, we also consider the direct-path selfinterference from the transmit array to the receive array. Moreover, Theorem 1 was derived using continuous linear arrays, but to make the simulation closer to practical implementations, we consider discrete arrays rather than continuous arrays.

As depicted in Fig. 8, the base station transmit array is a 36-element linear array, and likewise for the receive array. The separation between antenna elements within each array is $\Delta=\lambda / 2$, where $\lambda$ is the wavelength. These discrete arrays would therefore roughly correspond to a continuous arrays of length $L=18$ (normalized by wavelength). The transmit and receive arrays are parallel to each other and side-by-side, with a separation between the transmit and receive arrays of $5 \lambda$. We model the antenna elements as ideal point sources, such that the direct-path channel between transmit antenna $m$ and receive antenna $n$ can be simply modeled as $[38,39]$

$$
\left[\boldsymbol{C}_{\text {direct }}\right]_{n m}=\frac{e^{j k r_{n m}}}{r_{n m}}
$$

where, $r_{n m}$ is the distance between antennas $m$ and $n$, $k=\frac{2 \pi}{\lambda}$ is the wavenumber, and $j=\sqrt{-1}$. As is done in the simulation examples of [25], the backscattering is modeled via a simple discretization of the original continuous channel model

$$
\begin{aligned}
{\left[\boldsymbol{C}_{\text {scat }}\right]_{n m} } & =\frac{1}{\Delta} C_{12}\left(q_{n}, p_{m}\right) \\
& =\iint A_{R_{1}}\left(q_{n}, \tau\right) H_{12}(\tau, t) A_{T_{2}}\left(t, p_{n}\right) d \tau d t
\end{aligned}
$$

where $C_{12}$ is the continuous self-interference channel response described in Eqs. (2)-(8). We generate channel realizations by drawing $H(\tau, t)$ from a two-dimensional white gaussian process over $(\tau, t) \in \Psi_{R_{12}} \times \Psi_{T_{12}}$, and set $H(\tau, t)=0$ for $(\tau, t) \notin \Psi_{R_{12}} \times \Psi_{T_{12}}$. As in the symmetric-spread example, for convenience we let $\Psi_{T_{12}}=$ $\Psi_{R_{12}}=\Psi_{\text {Back. }}$. The total self-interference channel is $\boldsymbol{H}_{\text {self }}=\boldsymbol{C}_{\text {direct }}+\alpha \boldsymbol{C}_{\text {scat }}$, where $\alpha$ is a scalar chosen such that the backscattered self-interference is $20 \mathrm{~dB}$ weaker (on average) than the direct-path self-interference. We assume the noise floor is $80 \mathrm{~dB}$ below the transmit signal power. We consider the case of no backscattering, as well as cases where the backscattering subtends angles of $15^{\circ}, 45^{\circ}, 90^{\circ}$, and the fully backscattered case where the backscattering subtends $180^{\circ}$.

We simulate a transmit beamforming scheme inspired by the degrees-of-freedom achievability proof of section 3 . Let $d_{T}$ denote the dimension of the base stations transmit signal (i.e., the number of data streams the base station wishes to transmit). ${ }^{4}$ In the achievability proof, the base station transmitter avoids self-interference by projecting the $d_{T}$ transmit symbols onto the nullspace of the self-interference channel. Here, we generalize this nullspace-projection approach by having the base station transmitter project its $d_{T}$ transmit symbols onto the $d_{T}$ weakest singular vectors (i.e., the $d_{T}$ left singular vectors corresponding to the $d_{T}$ smallest singular values) of the self-interference channel, $\boldsymbol{H}_{\text {self. }}$ This beamforming approach, which we call "soft nulling" allows a flexible tradeoff between number of downlink dimensions, $d_{T}$, and the amount of self-interference generated: better self-interference suppression can be achieved by sacrificing transmit dimension. This concept of soft nulling is explored in depth in [27].

The results of the simulation are shown in Fig. 9. We see that for the case of no backscattering, the self-interference can be suppressed to the noise floor while maintaining a 32-dimensional downlink signal, only sacrificing 4 of the 36 downlink dimensions in order to suppress self-interference. However, in concurrence with the trend predicted by Theorem 1, as the angular spread of the backscattering increases, more transmit dimension must be sacrificed in order to suppress the self-interference to the noise floor. Merely increasing the backscattering spread to $15^{\circ}$ has a large impact: only 22 downlink transmit dimensions can be maintained while suppressing the self-interference to the noise floor-14 of the 36 transmit dimensions must be sacrificed to suppress the self-interference. In the case of a fully-backscattered self-interference channel, the self-interference cannot be
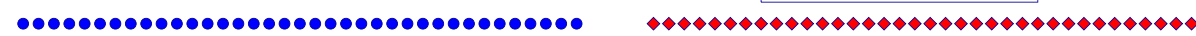

Fig. 8 Simulated base station array configuration. Both the transmit and receive arrays have 36 antennas with half-wavelength spacing between antenna elements, and 5-wavelength spacing between the transmit and receive arrays 


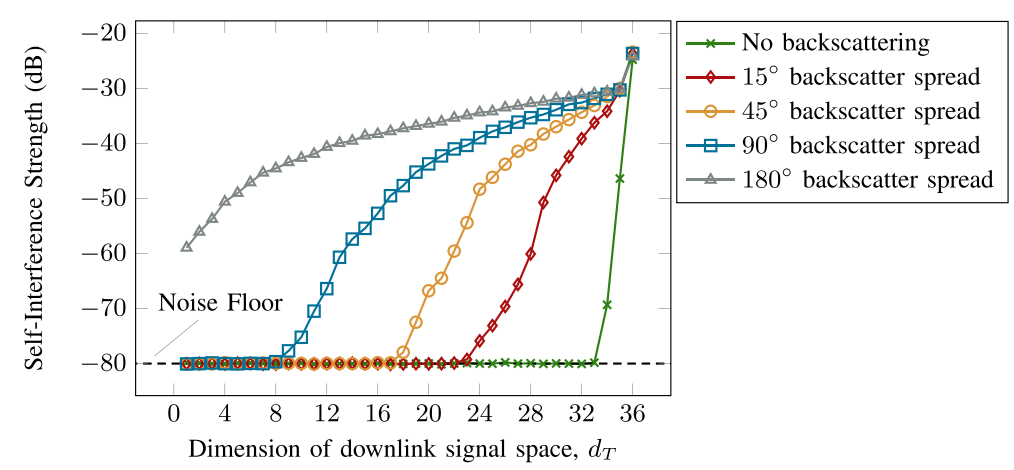

Fig. 9 Simulation results: self-interference versus dimension of downlink transmit signal

suppressed to the noise floor even if only one transmit dimension is used. In summary, we see that the angular spread of the backscattering dictates how many transmit dimensions must be sacrificed in order to sufficiently suppress self-interference.

\section{Conclusions}

Full-duplex operation presents an opportunity for base stations to as much as double their spectral efficiency by both transmitting downlink signal and receiving uplink signal at the same time in the same band. The challenge to full-duplex operation is high-powered self-interference that is received both directly from the base station transmitter and backscattered from nearby objects. The receiver can be spatially isolated from the transmitter by leveraging multi-antenna beamforming to avoid selfinterference, but such beamforming can also decrease the degrees-of-freedom of the intended uplink and downlink channels. We have leveraged a spatial antenna-theorybased channel model to analyze the spatial degreesof-freedom available to a full-duplex base station. The analysis has shown the full-duplex operation can indeed outperform half-duplex operation when either (1) the base station arrays are large enough for the base station to zero-force the backscattered self-interference or (2) the backscattering directions are not fully overlapped with the forward scattering directions, so that the base station can leverage the non-overlapped intervals for interference free signaling to/from the intended users.

\section{Endnotes}

${ }^{1}$ An additional challenge is the potential for the uplink user's transmission to interfere with the downlink user's reception, but in this paper we focus solely on the challenge of self-interference.

${ }^{2}$ We acknowledge that a continuous array which can support arbitrary current distributions may not be feasible to construct in practice due to the complications of feeding the array and achieving impedance match. However, as has been shown in the work of [24-26], a continuous array is nonetheless a very useful theoretical construct to develop performance bounds for any discrete antenna array subject to the same size constraint.

${ }^{3}$ There is extensive ongoing research on scheduling algorithms to select uplink and downlink users such that the uplink user generates little interference to the downlink user [40-44] (and references within). Thus, we make the simplifying assumption that there is no channel from the uplink transmitter, $T_{1}$, to the downlink receiver, $R_{2}$. This assumption allows the analysis to focus on the challenge of backscattered self-interference. An extension of this work, [45], which is outside the scope of this paper, focuses on the challenge of inter-user interference in a full-duplex network, and provides analysis for the case where there is a nonzero channel from $T_{1}$ to $R_{2}$.

${ }^{4}$ We call $d_{T}$ the "dimension of the transmit signal" instead of "degrees of freedom", because in this simulation, where SNR is finite, the term "degrees of freedom" is not correct by the rigorous definition used in the prior analysis.

\section{Appendix A: Functional analysis definitions}

Let $\mathcal{X}$ be a Hilbert space, the orthogonal complement of $\mathcal{S} \subseteq \mathcal{X}$, denoted $\mathcal{S}^{\perp}$, is the subset $\mathcal{S}^{\perp} \equiv\{x \in \mathcal{X}:\langle x, u\rangle=$ $0 \forall u \in \mathcal{S}$ \}. Let $\mathcal{X}$ and $\mathcal{Y}$ be vector spaces (e.g., Hilbert spaces) and let $\mathrm{C}: \mathcal{X} \rightarrow \mathcal{Y}$ be a linear operator. Let $\mathcal{S} \subseteq \mathcal{Y}$ be a subspace of $\mathcal{Y}$. The nullspace of $\mathrm{C}$, denoted $N(\mathrm{C})$, is the subspace $N(C) \equiv\{x \in \mathcal{X}: C x=0\}$. The range of $\mathrm{C}$, denoted $R(\mathrm{C})$, is the subspace $R(\mathrm{C}) \equiv\{\mathrm{C} x: x \in \mathcal{X}\}$. The preimage of $\mathcal{S}$ under $C, C^{\leftarrow}(\mathcal{S})$, is the subspace (one can check that if $\mathcal{S}$ is a subspace then $C^{\leftarrow}(\mathcal{S})$ is a subspace also). $C^{\leftarrow}(\mathcal{S}) \equiv\{x \in \mathcal{X}: C x \in \mathcal{S}\}$. The rank of $C$ is the dimension of the range of $C$. A fundamental result in functional analysis is that the dimension of the range of $C$ is 
also the dimension of the orthogonal complement of the nullspace of $C$ (i.e. the coimage of $C$ ) so that we can write $\operatorname{rank} \mathrm{C} \equiv \operatorname{dim} R(\mathrm{C})=\operatorname{dim} N(\mathrm{C})^{\perp}$.

\section{Appendix B: functional analysis lemmas}

Lemma 5 Let $\mathcal{X}$ and $\mathcal{Y}$ be Hilbert spaces and let $\mathrm{C}$ : $\mathcal{X} \rightarrow \mathcal{Y}$ be a compact linear operator. There exists a singular system $\left\{\sigma_{k}, v_{k}, u_{k}\right\}$, for $\mathrm{C}$ defined as follows. The set of functions $\left\{u_{k}\right\}$ form an orthonormal basis for $\overline{R(C)}$, the closure of the range of $\mathrm{C}$, and the set of functions $\left\{v_{k}\right\}$ form an orthonormal basis for $N(\mathrm{C})^{\perp}$, the coimage of $\mathrm{C}$. The set of positive real numbers $\sigma_{k}$, called the singular values of $\mathrm{C}$, are the nonzero eigenvalues of $\left(\mathrm{C}^{*} \mathrm{C}\right)$ arranged in decreasing order. The singular system diagonalizes $\mathrm{C}$ in the sense that for any $\left(\sigma_{k}, v_{k}, u_{k}\right) \in\left\{\sigma_{k}, v_{k}, u_{k}\right\}, C v_{k}=\sigma_{k} u_{k}$. Moreover, the operation of $C$ on any $x \in \mathcal{X}$ can be expanded as $\mathrm{C} x=\sum_{k} \sigma_{k}\left\langle x, v_{k}\right\rangle u_{k}$, which is called the singular value expansion of $\mathrm{C} x$. See Section 16.1 and 16.2 of [32] for a proof.

Lemma 6 Let $\mathcal{X}$ and $\mathcal{Y}$ be Hilbert spaces and let $\mathrm{C}$ : $\mathcal{X} \rightarrow \mathcal{Y}$ be a linear operator with closed range. There exists a unique linear operator $\mathrm{C}^{+}$, called the MoorePenrose pseudoinverse of $\mathrm{C}$, with the following properties: (i) $\mathrm{C}^{+} \mathrm{C} x=x \forall x \in N(\mathrm{C})^{\perp}$ (ii) $\mathrm{CC}^{+} y=y \forall y \in R$ (C) (iii) $R\left(\mathrm{C}^{+}\right)=N(\mathrm{C})^{\perp}$ (iv) $N\left(\mathrm{C}^{+}\right)=R(\mathrm{C})^{\perp}$.

See Definition 2.2 and Proposition 2.3 of [33] for a proof.

Lemma 7 Let $\mathcal{X}$ and $\mathcal{Y}$ be finite-dimensional Hilbert spaces and let $\mathrm{C}: \mathcal{X} \rightarrow \mathcal{Y}$ be a linear operator with closed range. Let $\mathcal{S} \subseteq \mathcal{Y}$ be a subspace of $\mathcal{Y}$. Then the dimension of the preimage of $\mathcal{S}$ under $C$ is

$$
\operatorname{dim} C^{\leftarrow}(\mathcal{S})=\operatorname{dim} N(\mathrm{C})+\operatorname{dim}(R(C) \cap \mathcal{S}) .
$$

Proof For notational convenience, let $d_{P} \equiv \operatorname{dim} C^{\leftarrow}(\mathcal{S})$, $d_{N} \equiv \operatorname{dim} N(\mathrm{C})$, and $d_{R \cap \mathcal{S}} \equiv \operatorname{dim}(R(\mathrm{C}) \cap \mathcal{S})$. Thus we wish to show that $d_{P}=d_{N}+d_{R \cap \mathcal{S}}$. First note that $N(\mathrm{C}) \subseteq \mathrm{C}^{\leftarrow}(\mathcal{S})$, since $\mathcal{S}$ is a subspace and hence contains the zero vector, and the preimage of the zero vector under $C$ is the nullspace of $C$. Denote the intersection between the preimage of $S$ under $C$ and the orthogonal complement of the nullspace of $C$ (i.e., the coimage) as

$$
\mathcal{B} \equiv C^{\leftarrow}(\mathcal{S}) \cap N(\mathrm{C})^{\perp}
$$

Note that $\mathcal{B}$ is a subspace of $\mathcal{X}$ since the intersection of any collection of subspaces is itself a subspace (see Thm. 1 on p. 3 of [46]). Every $x \in C^{\leftarrow}(\mathcal{S})$ can be expressed as $x=w+u$ for some $w \in N(C)$ and $u \in \mathcal{B}$, and $\langle w, u\rangle=0$ for any $w \in N(C)$ and $u \in \mathcal{B}$. Thus, we can say that the preimage, $C^{\leftarrow}(\mathcal{S})$, is the orthogonal direct sum of subspaces $N(C)$ and $\mathcal{B}$ ([32] Def. 4.26), a relationship we note we denote as $\mathrm{C}^{\leftarrow}(\mathcal{S})=N(\mathrm{C}) \oplus \mathcal{B}$.
Let $\left\{a_{i}\right\}_{i=1}^{d_{N}}$ be a basis for $N(\mathrm{C})$ and $\left\{b_{i}\right\}_{i=1}^{d_{\mathcal{B}}}$ be a basis for $\mathcal{B}$, where $d_{N}=\operatorname{dim} N(\mathrm{C})$ and $d_{\mathcal{B}}=\operatorname{dim} \mathcal{B}$. Construct the set $\left\{e_{i}\right\}_{i=1}^{d_{N}+d_{\mathcal{B}}}$ according to

$$
\left\{e_{i}\right\}_{i=1}^{d_{N}}=\left\{a_{i}\right\}_{i=1}^{d_{N}}, \quad\left\{e_{i}\right\}_{i=d_{N}+1}^{d_{N}+d_{\mathcal{B}}}=\left\{b_{i}\right\}_{i=1}^{d_{\mathcal{B}}} .
$$

We claim that $\left\{e_{i}\right\}_{i=1}^{d_{N}+d_{\mathcal{B}}}$ forms a basis for $\mathrm{C}^{\leftarrow}(\mathcal{S})$. To check that $\left\{e_{i}\right\}_{i=1}^{d_{N}+d_{\mathcal{B}}}$ is a basis for $\mathcal{C}^{\leftarrow}(\mathcal{S})$, we must first show $\left\{e_{i}\right\}_{i=1}^{d_{N}+d_{\mathcal{B}}}$ spans $\mathcal{C}^{\leftarrow}(\mathcal{S})$, and then show that the elements of $\left\{e_{i}\right\}_{i=1}^{d_{N}+d_{\mathcal{B}}}$ are linearly independent. Consider an arbitrary $x \in C^{\leftarrow}(\mathcal{S})$. Since $C^{\leftarrow}(\mathcal{S})=N(C) \oplus \mathcal{B}, x=w+u$ for some $w \in N(C)$ and $u \in \mathcal{B}$. Since by construction, $\left\{e_{i}\right\}_{i=1}^{d_{N}}$ is a basis for $N(\mathrm{C})$ and $\left\{e_{i}\right\}_{i=1+d_{N}}^{d_{N}+d_{\mathcal{B}}}$ is a basis for $N(\mathrm{C})$, one can choose $\lambda_{i}$ such that that $w=\sum_{i=1}^{d_{N}} \lambda_{i} e_{i}$ and $u=\sum_{i=1+d_{N}}^{d_{N}+d_{\mathcal{B}}} \lambda_{i} e_{i}$. Thus,

$$
x=w+v=\sum_{i=1}^{d_{N}} \lambda_{i} e_{i}+\sum_{i=1+d_{N}}^{d_{N}+d_{\mathcal{B}}} \lambda_{i} e_{i}=\sum_{i=1}^{d_{N}+d_{\mathcal{B}}} \lambda_{i} e_{i}
$$

for some $\lambda_{i}$. Thus $\left\{e_{i}\right\}_{i=1}^{d_{N}+d_{\mathcal{B}}}$ spans $\mathcal{C}^{\leftarrow}(\mathcal{S})$. Now let us show linear independence: that $\sum_{i=1}^{d_{N}+d_{\mathcal{B}}} \lambda_{i} e_{i}=0$ if and only if $\lambda_{i}=0$ for all $i \in\left\{1,2, \ldots, d_{N}+d_{\mathcal{B}}\right\}$. The "if" part is trivial, thus it remains to show that $\sum_{i=1}^{d_{N}+d_{\mathcal{B}}} \lambda_{i} e_{i}=0$ implies $\lambda_{i}=0 \forall i$. The condition $\sum_{i=1}^{d_{N}+d_{\mathcal{B}}} \lambda_{i} e_{i}=0$ implies

$$
\sum_{i=1}^{d_{N}} \lambda_{i} e_{i}=-\sum_{i=d_{N}+1}^{d_{N}+d_{\mathcal{B}}} \lambda_{i} e_{i}
$$

which implies $w=-u$ for some $w \in N(C)$ and $u \in \mathcal{B}$. Every element of $N(C)$ is orthogonal to every element of $\mathcal{B}$ by construction, hence the only way Eq. (129) can be satisfied is if $w=u=0$, that is if both sides of Eq. (129) are zero, implying $\lambda_{i}=0$ for all $i \in\left\{1,2, \ldots, d_{N}+d_{\mathcal{B}}\right\}$ as desired. Thus, we have shown $\left\{e_{i}\right\}_{i=1}^{d_{N}+d_{\mathcal{B}}}$ is a basis for $\mathrm{C}^{\leftarrow}(\mathcal{S})$, and hence

$$
d_{P}=d_{N}+d_{\mathcal{B}}
$$

Consider the set $\left\{C e_{i}\right\}_{i=1+d_{N}}^{d_{N}+d_{\mathcal{B}}}$. By the definition of range, each element of the set $\left\{C e_{i}\right\}_{i=1+d_{N}}^{d_{N}+d_{\mathcal{B}}}$ is in $R(\mathrm{C})$, and since by construction each $e_{i}$ is in $C^{\leftarrow}(\mathcal{S})$, each element of $\left\{C e_{i}\right\}_{i=1+d_{N}}^{d_{N}+d_{\mathcal{B}}}$ is also in $\mathcal{S}$. We therefore have that

$$
\operatorname{span}\left\{C e_{i}\right\}_{i=1+d_{N}}^{d_{N}+d_{\mathcal{B}}} \subseteq R(\mathrm{C}) \cap \mathcal{S},
$$

and since there are $d_{\mathcal{B}}$ elements in $\left\{C e_{i}\right\}_{i=1+d_{N}}^{d_{N}+d_{\mathcal{B}}}$, it must be that $d_{\mathcal{B}} \leq d_{R \cap \mathcal{S}}$. Substituting the above inequality into Eq. (130) gives $d_{P} \leq d_{N}+d_{R \cap \mathcal{S}}$. 
To complete the proof we must show that $d_{P} \geq d_{N}+$ $d_{R \cap \mathcal{S}}$. Let $\left\{s_{i}\right\}_{i=1}^{d_{R \cap \mathcal{S}}}$ be a basis for $R(\mathrm{C}) \cap \mathcal{S}$. By assumption $R(C)$ is closed, thus we have by Lemma 6 that the Moore-Penrose pseudoinverse, $\mathrm{C}^{+}$, exists and satisfies the properties listed in Lemma 6. Consider the set $\left\{\mathrm{C}^{+} s_{i}\right\}_{i=1}^{d_{R \cap \mathcal{S}}}$. We claim that

$$
\operatorname{span}\left\{\mathrm{C}^{+} s_{i}\right\}_{i=1}^{d_{R \cap \mathcal{S}}} \subseteq N(\mathrm{C})^{\perp} \cap \mathrm{C}^{\leftarrow}(\mathcal{S}) \equiv \mathcal{B} .
$$

By property (iv) in Lemma 6, we have that $\mathrm{C}^{+} s_{i} \in N(\mathrm{C})^{\perp}$ for each $\mathrm{C}^{+} s_{i} \in\left\{\mathrm{C}^{+} s_{i}\right\}_{i=1}^{d_{R \cap \mathcal{S}}}$. Since $s_{i} \in R(\mathrm{C})$, we have that $\mathrm{C}\left(\mathrm{C}^{+} s_{i}\right)=s_{i}$ by property (ii) of the pseudoinverse, and since $s_{i} \in \mathcal{S}$, we have that $\mathrm{CC}^{+} s_{i}=s_{i} \in \mathcal{S}$ for each $\mathrm{C}^{+} s_{i} \in$ $\left\{\mathrm{C}^{+} s_{i}\right\}_{i=1}^{d_{R \cap \mathcal{S}}}$. Thus, each element of $\left\{\mathrm{C}^{+} s_{i}\right\}_{i=1}^{d_{R \cap \mathcal{S}}}$ is also in $\mathrm{C}^{\leftarrow}(\mathcal{S})$, the preimage of $\mathcal{S}$ under $\mathrm{C}$. Thus we have that each element of $\left\{\mathrm{C}^{+} s_{i}\right\}_{i=1}^{d_{R \mathcal{S}}}$ is in $N(\mathrm{C})^{\perp} \cap \mathrm{C}^{\leftarrow}(\mathcal{S})$ which justifies the claim of Eq. (132). Now, Eq. (132) implies that $d_{R \cap \mathcal{S}} \leq d_{\mathcal{B}}$. Substituting the above inequality into Eq. (130) gives $d_{P} \geq d_{N}+d_{R \cap \mathcal{S}}$, concluding the proof.

Corollary 1 Let $\mathcal{X}$ and $\mathcal{Y}$ be finite-dimensional Hilbert spaces and let $\mathrm{C}: \mathcal{X} \rightarrow \mathcal{Y}$ be a linear operator with closed range. Let $\mathcal{S} \subseteq R(C) \subseteq \mathcal{Y}$ be a subspace of the range of $C$. Then, the dimension of the preimage of $\mathcal{S}$ under $\mathrm{C}$ is $\operatorname{dim} C^{\leftarrow}(\mathcal{S})=\operatorname{dim} N(\mathrm{C})+\operatorname{dim}(\mathcal{S})$.

Proof The proof follows trivially from Lemma 7 by noting that since $\mathcal{S} \subseteq R(\mathrm{C}), R(\mathrm{C}) \cap \mathcal{S}=\mathcal{S}$, which we substitute into Eq. 125 to obtain the corollary.

\section{Acknowledgements}

This work was partially supported by National Science Foundation (NSF) Grants CNS 0923479, CNS 1012921, CNS 1161596 and NSF Graduate Research Fellowship 0940902.

Received: 14 July 2016 Accepted: 26 November 2016

Published online: 15 December 2016

\section{References}

1. DW Bliss, PA Parker, AR Margetts, in Proceedings of the 2007 IEEE/SP 14th Workshop on Statistical Signal Processing. Simultaneous transmission and reception for improved wireless network performance (Institute of Electrical and Electronics Engineers (IEEE), New York, 2007), pp. 478-482

2. AK Khandani. Methods for spatial multiplexing of wireless two-way channels, (2010). US Patent US 7817641 B1. https://www.google.com/ patents/US7817641

3. B Radunovic, D Gunawardena, P Key, APN Singh, V Balan, G Dejean, Rethinking indoor wireless: low power, low frequency, full duplex (2009). Microsoft Research, Technical Report \# MSR-TR-2009-148, https://www. microsoft.com/en-us/research/publication/rethinking-indoor-wirelesslow-power-low-frequency-full-duplex/

4. M Duarte, A Sabharwal, in Proc. 2010 Asilomar Conference on Signals and Systems. Full-duplex wireless communications using off-the-shelf radios: feasibility and first results (Institute of Electrical and Electronics Engineers (IEEE), New York, 2010)

5. J Choi, M Jain, K Srinivasan, P Levis, S Katti, in MobiCom 2010. Achieving single channel, full duplex wireless communication (Association of Computing Machinery (ACM) Publications, New York, 2010)

6. M Jain, Jl Choi, T Kim, D Bharadia, S Seth, K Srinivasan, P Levis, S Katti, P Sinha, in MobiCom 2011. Practical, real-time, full duplex wireless (New York, 2011), pp. 301-312. http://doi.acm.org/10.1145/2030613.2030647
7. M Duarte, C Dick, A Sabharwal, Experiment-driven characterization of full-duplex wireless systems. IEEE Trans. Wireless Commun. 11(12) 4296-4307 (2012)

8. A Sahai, G Patel, A Sabharwal, Pushing the limits of full-duplex: design and real-time implementation (2011). Rice Univeristy, Technical Report \# TREE1104. https://arxiv.org/abs/1107.0607

9. MA Khojastepour, K Sundaresan, S Rangarajan, X Zhang, S Barghi, in ACM 1480 Workshop on Hot Topics in Networks. The case for antenna cancellation for scalable full-duplex wireless communications (ACM, New York, 2011), pp. 17:1-17:6

10. E Aryafar, MA Khojastepour, K Sundaresan, S Rangarajan, M Chiang, in 1483 Proceedings of the 18th annual international conference on Mobile 1484 computing and networking. MIDU: enabling MIMO full duplex, ser. 1485 Mobicom '12 (ACM, New York, 2012), pp. 257-268

11. M Duarte, A Sabharwal, V Aggarwal, R Jana, K Ramakrishnan, C Rice, N Shankaranarayanan, Design and characterization of a full-duplex multiantenna system for Wi-Fi networks. IEEE Trans. Vehicular Technol. 63(3), 1160-1177 (2014)

12. M Duarte, Full-duplex wireless: Design, implementation and characterization (2012). Ph.D.dissertation, Rice University. http://warp.rice. edu/trac/wiki/DuartePhDThesis

13. TRiihonen, S Werner, R Wichman, in Wireless Communications and Networking Conference, WCNC 2009. Comparison of full-duplex and halfduplex modes with a fixed amplify-and-forward relay (IEEE, 2009) (Institute of Electrical and Electronics Engineers (IEEE), New York, 2009), pp. 1-5

14. T Riihonen, S Werner, R Wichman, Z Eduardo, in IEEE 10th Workshop on Signal Processing Advances in Wireless CommunicationsCommunications, 2009. SPAWC '09. On the feasibility of full-duplex relaying in the presence of loop interference (Institute of Electrical and Electronics Engineers (IEEE), New York, 2009), pp. 275-279

15. B Day, A Margetts, D Bliss, P Schniter, Full-duplex bidirectional MIMO: achievable rates under limited dynamic range. IEEE Trans. Signal Process. 60(7), 3702-3713 (2012)

16. B Day, A Margetts, D Bliss, P Schniter, Full-duplex MIMO relaying: achievable rates under limited dynamic range. IEEE J. Selected Areas Commun. 30(8), 1541-1553 (2012)

17. T Riihonen, R Wichman, J Hamalainen, in IEEE International Symposium on Wireless Communication Systems. 2008. ISWCS '08. Co-phasing full-duplex relay link with non-ideal feedback information (Institute of Electrical and Electronics Engineers (IEEE), New York, 2008), pp. 263-267

18. T Riihonen, S Werner, J Cousseau, R Wichman, in 42nd Asilomar Conference on Signals, Systems and Computers, 2008. Design of co-phasing allpass filters for fullduplex OFDM relays (Institute of Electrical and Electronics Engineers (IEEE), New York, 2008), pp. 1030-1034

19. T Riihonen, S Werner, R Wichman, Mitigation of loopback self-interference in full-duplex MIMO relays. IEEE Trans. Signal Process. 59(12), 5983-5993 (2011)

20. E Everett, M Duarte, C Dick, A Sabharwal, in Asilomar Conference on Signals, Systems and Computers. Empowering full-duplex wireless communication by exploiting directional diversity (Institute of Electrical and Electronics Engineers (IEEE), New York, 2011)

21. E Everett, A Sahai, A Sabharwal, Passive self-interference suppression for full-duplex infrastructure nodes. IEEE Trans. Wireless Commun. 13(2), 680-694 (2014)

22. A Sahai, G Patel, C Dick, A Sabharwal, On the impact of phase noise on active cancelation in wireless full-duplex. IEEE Trans. Vehicular Technol. 62(9), 4494-4510 (2013)

23. A Sabharwal, P Schniter, D Guo, DW Bliss, S Rangarajan, R Wichman, In-band full-duplex wireless: Challenges and opportunities. IEEE Journal on Selected Areas in Communications. 32(9), 1637-1652 (2014)

24. A Poon, R Brodersen, D Tse, Degrees of freedom in multiple-antenna channels: a signal space approach. IEEE Trans. Inf. Theory. 51(2), 523-536 (2005)

25. A Poon, D Tse, R Brodersen, Impact of scattering on the capacity, diversity, and propagation range of multiple-antenna channels. IEEE Trans. Inf. Theory. 52(3), 1087-1100 (2006)

26. A Poon, D Tse, Degree-of-freedom gain from using polarimetric antenna elements. IEEE Trans. Inf. Theory. 57(9), 5695-5709 (2011)

27. E Everett, C Shepard, L Zhong, A Sabharwal, SoftNull: many-antenna full-duplex wireless via digital beamfoming. IEEE Trans. Wireless Commun. 15(12), 8077-8092 (2016) 
28. A Poon, M Ho, in IEEE International Conference on Communications, 2003. ICC'03. Indoor multiple-antenna channel characterization from 2 to 8 $\mathrm{GHz}$, vol 5 (Institute of Electrical and Electronics Engineers (IEEE), New York, 2003), pp. 3519-3523

29. Q Spencer, B Jeffs, M Jensen, A Swindlehurst, Modeling the statistical time and angle of arrival characteristics of an indoor multipath channel. IEEE J. Selected Areas Commun. 18(3), 347-360 (2000)

30. RJ-M Cramer, An evaluation of ultra-wideband propagation channels (2000). Ph.D. dissertation, University of Southern California

31. R Heddergott, P Truffer, Statistical characteristics of indoor radio propagation in NLOS scenarios. European Cooperation in the field of scientific and technical research, Valencia, Spain, In COST. 259, 1-15 (2000)

32. N Young, An Introduction to Hilbert Space. (Cambridge University Press, Cambridge, 1988)

33. HW Engl, M Hanke, A Neubauer, Regularization of Inverse Problems. (Kluwer Academic Publishers, Dordrecht, 1996)

34. E Telatar, Capacity of multi-antenna gaussian channels. European Trans. Telecommu. 10(6), 585-595 (1999)

35. L Ke, Z Wang, Degrees of freedom regions of two-user MIMO $Z$ and full interference channels: the benefit of reconfigurable antennas. IEEE Trans. Inf. Theory. 58(6), 3766-3779 (2012)

36. S Jafar, M Fakhereddin, Degrees of freedom for the MIMO interference channel. IEEE Trans. Inf. Theory. 53(7), 2637-2642 (2007)

37. S Krishnamurthym, S Jafar, in 2012 IEEE Global Communications Conference (GLOBECOM). Degrees of freedom of 2-user and 3-user rank-deficient MIMO interference channels (Institute of Electrical and Electronics Engineers (IEEE), New York, 2012), pp. 2462-2467

38. DNC Tse, P Viswanath, Fundamentals of Wireless Communication. (Cambridge University Press, Cambrige, 2005)

39. CA Balanis, Antenna Theory: Analysis and Design, 3rd ed. (Wiley-Interscience, Hoboken, 2005)

40. A Tang, X Wang, A-Duplex: Medium access control for efficient coexistence between full duplex and half duplex communications. IEEE Trans. Wireless Commun. 14(10), 15871-58851 (2015)

41. JY Kim, O Mashayekhi, H Qu, M Kazadiieva, P Levis, JANUS: a novel MAC protocol for full duplex radio. Stanford Univerisity, Tech. Rep. CSTR. 2.7(23) (2013). http://hci.stanford.edu/cstr/reports/2013-02.pdf

42. N Singh, D Gunawardena, A Proutiere, B Radunovic, H Balan, P Key, in Modeling and Optimization in Mobile, Ad Hoc and Wireless Networks (WiOpt), 2011. Efficient and fair MAC for wireless networks with self-interference cancellation (Institute of Electrical and Electronics Engineers (IEEE), New York, 2011), pp. 94-101

43. Q Gao, G Chen, L Liao, Y Hua, in Computing, Networking and Communications (ICNC), 2014 International Conference on. Full-duplex cooperative transmission scheduling in fast-fading MIMO relaying wireless networks (Institute of Electrical and Electronics Engineers (IEEE), New York, 2014), pp. 771-775

44. C Karakus, SN Diggavi, Opportunistic scheduling for full-duplex uplink-downlink networks (2015). CoRR, vol. abs/1504.05898. http://arxiv. org/abs/1504.05898

45. Y Chen, A Sabharwal, Degrees of freedom of spatial self-interference suppression for in-band full-duplex with inter-node interference (2016). arXiv preprint arXiv: 1606.05809. https://arxiv.org/pdf/1606.05809.pdf

46. PD Lax, Funtional Analysis. (Wiley, New York, 2002)

\section{Submit your manuscript to a SpringerOpen ${ }^{\circ}$ journal and benefit from:}

- Convenient online submission

- Rigorous peer review

- Immediate publication on acceptance

- Open access: articles freely available online

- High visibility within the field

- Retaining the copyright to your article

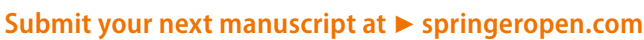

